University of South Florida

DIGITAL COMMONS

Digital Commons @ University of

@ UNIVERSITY OF SOUTH FLORIDA

South Florida

9-1994

\title{
Equatorially Trapped Waves of a Coupled Ocean-Atmosphere System
}

\author{
Chunzai Wang \\ University of South Florida \\ Robert $\mathrm{H}$. Weisberg \\ University of South Florida, weisberg@marine.usf.edu
}

Follow this and additional works at: https://digitalcommons.usf.edu/msc_facpub

Part of the Marine Biology Commons

\section{Scholar Commons Citation}

Wang, Chunzai and Weisberg, Robert H., "Equatorially Trapped Waves of a Coupled Ocean-Atmosphere System" (1994). Marine Science Faculty Publications. 146.

https://digitalcommons.usf.edu/msc_facpub/146

This Article is brought to you for free and open access by the College of Marine Science at Digital Commons @ University of South Florida. It has been accepted for inclusion in Marine Science Faculty Publications by an authorized administrator of Digital Commons @ University of South Florida. For more information, please contact digitalcommons@usf.edu. 


\title{
Equatorially Trapped Waves of a Coupled Ocean-Atmosphere System
}

\author{
Chunzal Wang and Robert H. WeISBERG \\ Department of Marine Science, University of South Florida, St. Petersburg, Florida
}

(Manuscript received 17 May 1993, in final form 23 January 1994)

\begin{abstract}
Equatorially trapped waves of a simplified coupled ocean-atmosphere system are described by applying the formalism for conventional equatorially trapped waves with the assumption that the zonal wind stress and sea surface temperature perturbations are proportional. In this system, inertial-gravity and Rossby-gravity waves are unaffected by coupling whereas Rossby and Kelvin waves are affected, and in the low-frequency limit, these Rossby and Kelvin waves transform to slow westward and eastward propagating wave modes, respectively. The primary modifications by air-sea coupling are a decrease in phase speed and an increase in meridional scale. The properties of these coupled waves are useful in discussing several features, observed and modeled, relative to the evolution of the El Niño-Southern Oscillation.
\end{abstract}

\section{Introduction}

Two paradigms have been developed for interpreting El Niño-Southern Oscillation (ENSO)-related, coupled ocean-atmosphere numerical models: 1) the delayed oscillator mechanism of Suarez and Schopf (1988) and Battisti and Hirst (1989) and 2) the slow thermal or sea surface temperature (SST) mode mechanism of Hirst $(1986,1988)$ and Neelin (1991). The delayed oscillator mechanism depends upon the propagation of conventional equatorially trapped ocean Kelvin and Rossby waves, along with localized oceanatmosphere coupling, in setting the ENSO timescale. The slow mode mechanism argues for a slowly propagating, coupled mode that is distinctly different from the conventional equatorial waves and for which the propagation time across the basin sets the ENSO timescale. The developments leading to these paradigms are reviewed by McCreary and Anderson (1991).

The present paper investigates the properties of equatorially trapped waves of a coupled ocean-atmosphere system, simplified to use the same formalism as Matsuno (1966) for the case of conventional equatorially trapped waves. Motivation follows from several features, either observed in the ocean or arising in the hierarchy of ENSO-related numerical models. First, all numerical models of the coupled ocean-atmosphere system, from the simplest linear perturbation models (e.g., Hirst 1986, 1988) to the most complicated general circulation models (e.g., Barnett et al. 1991; Chao and

Corresponding author address: Dr. Robert $\mathrm{H}$. Weisberg, Dept. of Marine Science, University of South Florida, 140 Seventh Avenue South, St. Petersburg, FL 33701-5016.
Philander 1993) show meridional scales for coupled oscillations larger than the ocean's Rossby radius of deformation. This broadening of meridional scale with respect to ENSO is also an observed property of the ocean thermocline (e.g., White et al. 1987, 1989; Kessler 1990). Second, in the Kessler (1990) analysis these thermocline displacements, found to be maximum at about $12^{\circ} \mathrm{N}$, propagated westward, but at speeds slower than the gravest mode equatorially trapped Rossby wave speed. Third, the role of these so-called off-equatorial Rossby waves in the evolution of the ENSO cycle has been controversial, with Graham and White (1991) contending that they are important and Kessler (1991), Battisti (1991), and Wakata and Sarachik (1991) contending that they are not. Fourth, the mechanisms at work in coupled GCM simulations of ENSO remain unresolved. For example, similar El Niño and La Niña states are achieved when using either high- or low-resolution ocean model components (Philander et al. 1992; Lau et al. 1992) despite the fact that the lowresolution model cannot resolve conventional equatorial waves and the authors' contention that the highresolution model shows no evidence for them. Fifth, with conventional equatorially trapped Kelvin waves having been observed in the oceans (e.g., Knox and Halpern 1982; Katz 1987) and having been used, along with Rossby waves, in describing the seasonal evolution of the Atlantic Ocean's equatorial thermocline (e.g., Weisberg and Tang 1990), a question arises regarding the conditions, if any, by which conventional equatorially trapped waves are modified by ocean-atmosphere coupling.

The paper proceeds as follows. Section 2 formulates the problem by assuming that the perturbations in zonal wind stress and sea surface temperature are proportional, gives the general solution, the solution for 
Kelvin waves as a special case when the meridional velocity component is zero, and describes the full dispersion relationship for the equatorially trapped waves of this simplified coupled ocean-atmosphere system. Section 3 discusses the scale modifications and describes the eigenfunctions for the coupled Rossby (Kelvin) and westward (eastward) propagating slow mode waves, comparing these eigenfunctions with their uncoupled ocean counterparts. Section 4 then provides discussion on these findings relative to the model limitations and the motivating factors.

\section{Coupled ocean-atmosphere equatorial wave modes}

\section{a. Problem formulation}

Most models of the coupled ocean-atmosphere system relative to ENSO have considered the ocean to be driven by the surface winds and the atmosphere to be driven by SST-induced heating. Consistent with this, we seek the simplest set of equations, solvable analytically, for the purpose of gaining insight into the properties of coupled ocean-atmosphere equatorial waves. The ocean dynamical equations are those of a linear, equatorial $\beta$-plane reduced-gravity model, perturbed about a basic state of rest by the zonal component of surface wind stress $\tau^{x}$. The upper layer has density $\rho$ and mean depth $H_{0}$; the lower layer has slightly higher density and is infinitely deep and motionless. The interface between these layers represents the tropical thermocline, separating the warm near surface waters from the cold deep waters. The ocean thermodynamics controlling the variations in the sea surface temperature anomaly ( $T$ ) consist of a trade-off between ocean processes and surface heat fluxes. The ocean processes are lumped into a term proportional to the upper-layer thickness perturbation except for zonal advection by the perturbation currents on a mean temperature gradient, which is treated separately. Thus, the equations governing the momentum, mass, and SST anomalies are

$$
\begin{gathered}
\frac{\partial u}{\partial t}-\beta y v=-g^{\prime} \frac{\partial h}{\partial x}+\frac{\tau^{x}}{\rho H_{0}}-\gamma u \\
\frac{\partial v}{\partial t}+\beta y u=-g^{\prime} \frac{\partial h}{\partial y}-\gamma v \\
\frac{\partial h}{\partial t}+H_{0}\left(\frac{\partial u}{\partial x}+\frac{\partial v}{\partial y}\right)=-\gamma h \\
\frac{\partial T}{\partial t}+\eta u=\sigma h-\gamma T
\end{gathered}
$$

where $u$ and $v$ are the velocity components in the zonal $(x)$ and meridional $(y)$ coordinate directions; $h$ is the upper-layer thickness perturbation, $t$ is time, $g^{\prime}$ the reduced gravity, $\beta$ the gradient in planetary vorticity, $\sigma$ the warming parameter, $\eta$ the mean zonal temperature gradient, and $\gamma$ is a coefficient representing Rayleigh friction, Newtonian cooling, and thermal damping, thus providing dissipation in the momentum, mass, and SST equations, respectively. The thermodynamic equation is similar in form to the Hirst (1986) model III. It is recognized as being overly simplistic since, with constant parameters, it omits background state processes that may also be important.

To form a coupled ocean-atmosphere system, we need a way of specifying $\tau^{x}$ in terms of ocean variables. The zonal asymmetry in wind velocity that occurs near the equator (due to the $\beta$ effect) in reduced gravity, SST-forced atmosphere models such as Gill (1980) suggests a relationship between the anomalies of $\tau^{x}$ and SST. Several authors have used this concept. Battisti and Hirst (1989), in their derivation of the delayed oscillator equation, assumed $\tau^{x}$ proportional to SST based on numerical model results averaged over the eastern equatorial Pacific. Schopf and Suarez (1990) assumed $\tau^{x}$ to have a specified meridional structure with magnitude proportional to SST at a fixed point on the equator. Cane et al. (1990) related $\tau^{x}$ to the thermocline height anomaly at the eastern boundary, also with specified meridional structure, and Neelin (1991) formulated a more general proportionality relation. By assuming $\tau^{x}$ and SST to be proportional everywhere, Wang and Weisberg (1994) obtained a solution for eastward propagating wave modes. These waves show either exponential growth or decay depending upon whether or not there is a zonal phase difference between $\tau^{x}$ and SST. By omitting the phase lag the neutral wave results may be obtained over the full range of equatorially trapped waves. Thus, we seek solutions of the form

$$
\left(\begin{array}{c}
u(x, y, t) \\
v(x, y, t) \\
h(x, y, t) \\
T(x, y, t) \\
\tau^{x}(x, y, t)
\end{array}\right)=\left(\begin{array}{c}
u(y) \\
v(y) \\
h(y) \\
T(y) \\
\rho \mu H_{0} T(y)
\end{array}\right) e^{i(k x-\omega t)-\gamma t}
$$

where $\mu$ is the ocean-atmosphere coupling coefficient, $k$ is the zonal wavenumber component, and $\omega$ is the frequency. With $\omega$ taken to be positive, the direction of zonal phase propagation is determined by the sign of $k$. The assumption of equal values for Rayleigh friction, Newtonian cooling, and thermal damping allows the effects of dissipation to be factored out, resulting in $₹$ neutral wave dispersion relationship and an evanescent factor $e^{-\gamma t}$ multiplying the eigenfunctions. The limitations imposed by the three principal assumptions: 1) proportionality between $\tau^{x}$ and SST, 2) spatial homogeneity in coupling and other parameters, and 3 ) dissipation by equal values of Rayleigh friction, Newtonian cooling, and thermal damping will be discussed in section 4 .

\section{b. General solution}

Substituting Eq. (2.1.5) into Eqs. (2.1.1-4) yields four equations with four unknowns $u, v, h$, and $T$ : 


$$
\begin{gathered}
-i \omega u-\beta y v=-i k g^{\prime} h+\mu T \\
-i \omega v+\beta y u=-g^{\prime} \frac{d h}{d y} \\
-i \omega h+i k H_{0} u+H_{0} \frac{d v}{d y}=0 \\
-i \omega T+\eta u=\sigma h .
\end{gathered}
$$

Eliminating $u, h$, and $T$ results in the single equation for $v$ :

$$
\frac{d^{2} v}{d y^{2}}-A y \frac{d v}{d y}+\left(B-D^{2} y^{2}\right) v=0
$$

where

$$
\begin{gathered}
A \equiv \frac{H_{0} \beta \mu \sigma}{c^{2}\left(\omega^{2}-\mu \eta\right)}, \\
D^{2} \equiv \frac{\beta^{2} \omega^{2}}{c^{2}\left(\omega^{2}-\mu \eta\right)}, \\
B \equiv \frac{\omega^{2}}{c^{2}}+\frac{H_{0} k \mu \sigma \omega}{c^{2}\left(\omega^{2}-\mu \eta\right)}-\frac{k^{2} \omega^{2}}{\omega^{2}-\mu \eta}-\frac{k \beta \omega}{\omega^{2}-\mu \eta},
\end{gathered}
$$

and $c=\left(g^{\prime} H_{0}\right)^{1 / 2}$ is the ocean equatorial Kelvin wave speed. Equation (2.2.5) may be transformed into canonical form using the substitution

$$
v(y)=\tilde{v}(\xi) \exp \left[-\frac{\xi^{2}}{2}\left(1-\frac{A L^{2}}{2}\right)\right],
$$

whence

$$
\frac{d^{2} \tilde{v}}{d \xi^{2}}-2 \xi \frac{d \tilde{v}}{d \xi}+\left[L^{2}\left(B+\frac{A}{2}\right)-1\right] \tilde{v}=0,
$$

where $\xi \equiv y / L$, and $L \equiv\left(A^{2} / 4+D^{2}\right)^{-1 / 4}$. Equation (2.2.10) is the Hermite equation. Its solutions satisfy the boundary conditions, $v(y) \rightarrow 0$ as $y \rightarrow \pm \infty$, when the constant $L^{2}(B+A / 2)$ equals an odd integer. This results in the dispersion relationship

$$
\begin{aligned}
& \frac{2 c^{2}\left(\omega^{2}-\mu \eta\right)}{\beta \sqrt{4 \omega^{2} c^{2}\left(\omega^{2}-\mu \eta\right)+H_{0}^{2} \mu^{2} \sigma^{2}}} \\
& \times\left[\frac{\omega^{2}}{c^{2}}-\frac{k^{2} \omega^{2}}{\omega^{2}-\mu \eta}-\frac{k \beta \omega}{\omega^{2}-\mu \eta}\right. \\
& \left.+\frac{H_{0} \mu \sigma}{c^{2}\left(\omega^{2}-\mu \eta\right)}\left(k \omega+\frac{\beta}{2}\right)\right]=2 n+1, \\
& n=0,1,2, \ldots
\end{aligned}
$$

and the solution

$$
\begin{aligned}
v(y) & =C_{1} N_{n} \exp \left(-\frac{y^{2}}{2 L_{R}^{2}}\right) H_{n}(y / L) \\
& =C_{1} \psi_{n}(\xi)
\end{aligned}
$$

where $C_{1}$ is a dimensional constant, $H_{n}(\xi)$ is the Hermite polynomial of order $n, N_{n}=\left(2^{n} n ! \pi^{1 / 2}\right)^{-1 / 2}$ is the normalization factor for $\psi_{n}(\xi)$, and $L_{R}=L /\left(1-A L^{2} /\right.$ $2)^{1 / 2}$. The eigenfunctions $\psi_{n}(\xi)=N_{n} \exp \left(-\xi^{2} / 2\right.$ $\left.+A L^{2} \xi^{2} / 4\right) H_{n}(\xi)$ corresponding to the eigenvalues of Eq. (2.2.5) are orthonormal over the interval $-\infty<\xi$ $<\infty$ with respect to the weighting function $r(\xi)$ $=\exp \left(-A L^{2} \xi^{2} / 2\right)$; that is,

$$
\begin{aligned}
\int_{-\infty}^{+\infty} & r(\xi) \psi_{m}(\xi) \psi_{n}(\xi) d \xi \\
\quad= & \int_{-\infty}^{+\infty} N_{m} N_{n} e^{-\xi^{2}} H_{m}(\xi) H_{n}(\xi) d \xi=\delta_{m n},
\end{aligned}
$$

where $\delta_{m n}=1$, if $m=n$, and $\delta_{m n}=0$, if $m \neq n$ (see appendix ).

If the ocean is not coupled with the atmosphere ( $\mu$ $=0)$, then $A=0, L_{R}=L=(c / \beta)^{1 / 2}$, and (2.2.11) reduces to the conventional dispersion relationship for equatorially trapped waves of either the ocean or the atmosphere (Matsuno 1966):

$\frac{c}{\beta}\left(\frac{\omega^{2}}{c^{2}}-k^{2}-\frac{k \beta}{\omega}\right)=2 n+1, \quad n=0,1,2, \cdots$,

which includes inertial-gravity and Rossby waves, along with Rossby-gravity and Kelvin waves as special cases. Analogous with these conventional equatorially trapped waves, the complete dispersion relationship (2.2.11) includes ocean-atmosphere coupled inertialgravity, Rossby, Rossby-gravity, Kelvin, and a modified class of Rossby and Kelvin waves at low frequency referred to as slow mode waves. The effects of the ocean-atmosphere coupling upon these equatorially trapped waves vary inversely with frequency. Thus, the coupled inertial-gravity and Rossby-gravity waves are very similar to their conventional counterparts. For frequencies intermediately between the Rossby-gravity and the slow mode waves (that is, $\omega^{2}$ small, but much larger than $\mu \eta) L^{2} \approx c / \beta$ and the dispersion relationship for the coupled Rossby waves may be approximated by

$$
\omega=\frac{1}{2} \omega_{c}\left[1+\left(1-\frac{2 H_{0} \mu \sigma}{c^{2} k \omega_{c}}\right)^{1 / 2}\right],
$$

where

$$
\omega_{c} \equiv-\frac{k \beta}{k^{2}+(2 n+1) \beta / c}
$$

is the conventional dispersion relationship for the equatorially trapped Rossby waves. At lower frequency, where terms quadratic or higher in $\omega$ may be neglected, the dispersion relationship (2.2.11) reduces to

$\frac{2 c^{2}}{\beta H_{0} \mu \sigma}\left(-k \beta \omega+\frac{H_{0} \mu \sigma k \omega}{c^{2}}+\frac{H_{0} \mu \sigma \beta}{2 c^{2}}\right)=2 n+1$, 
from which

$$
\omega=-\frac{n H_{0} \mu \sigma \beta}{\left(c^{2} \beta-H_{0} \mu \sigma\right) k} \approx-\frac{n H_{0} \mu \sigma}{c^{2} k} .
$$

Equation (2.2.18) is the approximate dispersion relationship for a westward propagating slow mode wave, shown later to be a continuation of the coupled Rossby wave dispersion relationship at low frequency.

\section{c. Special solution}

Similar to conventional equatorially trapped waves, we seek a solution with the meridional velocity component identically zero. With $v(y) \equiv 0$, Eqs. (2.2.14) are

$$
\begin{gathered}
-i \omega u=-i k g^{\prime} h+\mu T \\
\beta y u=-g^{\prime} \frac{d h}{d y} \\
-i \omega h+i k H_{0} u=0 \\
-i \omega T+\eta u=\sigma h .
\end{gathered}
$$

This set of algebraic equations has solutions for $u, h$, and $T$ only when

$$
\omega^{2}+\frac{H_{0} \mu \sigma k}{\omega}-k^{2} c^{2}-\mu \eta=0
$$

which is the dispersion relationship for this special case. Combining Eqs. (2.3.2) and (2.3.3) leads to the solution

$$
h(y)=C_{2} \exp \left[-\frac{\beta \omega}{2 k c^{2}} y^{2}\right],
$$

where $C_{2}$ is a dimensional constant. Note that the meridional length scale, which we will refer to as $L_{K}$, is given by $L_{K}=\left(k c^{2} / \beta \omega\right)^{1 / 2}$.

The dispersion relationship, being cubic in $\omega$, has three roots. It is anticipated that one of the these roots should correspond to the coupled Kelvin wave, and one to the coupled slow mode wave. At relatively high frequencies the dispersion relationship reduces to

$$
\omega=\sqrt{k^{2} c^{2}+\mu \eta}
$$

and similar to the conventional Kelvin wave, the negative root is rejected because the associated wave amplitudes for $u, h$, and $T$ increase exponentially with $y$. At frequencies low enough so that the second-order term in $\omega$ can be neglected, Eq. (2.3.5) reduces to

$$
\omega=\frac{H_{0} \mu \sigma k}{c^{2} k^{2}+\mu \eta},
$$

which is the dispersion relationship for an eastward propagating slow mode wave. Furthermore, for $\mu \eta$ $\ll c^{2} k^{2}$, Eq. (2.3.8) becomes

$$
\omega=\frac{H_{0} \mu \sigma}{c^{2} k},
$$

which is the same as the dispersion relationship for the westward propagating slow mode wave, given by Eq. (2.2.18), with $n$ set equal to -1 (conventional for equatorially trapped waves). Thus, both the coupled Rossby and Kelvin waves have westward and eastward propagating slow mode waves, respectively, in their low-frequency limits.

\section{d. The complete dispersion relationship}

A comparison between the dispersion relationships for the ocean-atmosphere coupled and the conventional ocean equatorially trapped waves is given in Figs. $1 \mathrm{a}$ and $1 \mathrm{~b}$. Only the Rossby, Kelvin, and slow mode waves are considered since the Rossby-gravity and the inertial-gravity waves are essentially unchanged by coupling, owing to their frequency range. Except at low frequency there is very little difference between the conventional and the coupled waves. However, once the physics of the coupling begins to dominate over the intrinsic $\beta$-plane ocean wave propagation physics, the character of the dispersion relationship changes and a continuous transition occurs between Rossby (Kelvin) waves and westward (eastward) propagating slow mode waves. Since the local time derivatives are the only terms within this model yielding propagation, the phase speed for the coupled waves must decrease with decreasing frequency as these derivative terms become less important than the coupling term [using a different set of assumptions Lau (1981) also noted a reduction in phase speed for a coupled Kelvin wave]. As the phase speed decreases with decreasing frequency, the zonal wavenumber magnitude must either increase or remain constant (for the case $\sigma=0$ ). Where this transition occurs in the dispersion plane depends upon the parameters of the coupled system.

The parameters used in Figs. $1 \mathrm{a}$ and $1 \mathrm{~b}$ are $\mu=0.85$ $\times 10^{-7} \mathrm{~m} \mathrm{~s}^{-2} \mathrm{~K}^{-1}, \sigma=5.0 \times 10^{-9} \mathrm{~K} \mathrm{~m}^{-1} \mathrm{~s}^{-1}, \eta$ $=-5.0 \times 10^{-7} \mathrm{~K} \mathrm{~m}^{-1}, H_{0}=200 \mathrm{~m}, c=2 \mathrm{~m} \mathrm{~s}^{-1}$, and $\beta=2.29 \times 10^{-11} \mathrm{~m}^{-1} \mathrm{~s}^{-1}$. With these values, the warming and zonal advection terms are the same order (tending about $0.15 \mathrm{~K} \mathrm{mo}^{-1}$ for $h$ and $u$ perturbations of $10 \mathrm{~m}^{2}$ and $0.1 \mathrm{~m} \mathrm{~s}^{-1}$, respectively) and the coupling coefficient results in an approximate $0.02 \mathrm{~N} \mathrm{~m}^{-2}$ wind stress perturbation per each $1 \mathrm{~K}$ perturbation in SST. Modifications to the Rossby and Kelvin waves become discernible between frequencies of 10-20 $\mathrm{rad} \mathrm{yr}^{-1}$. As frequency decreases the effects of coupling impacts the higher mode Rossby waves first with the gravest mode being modified the least. The transition to slow mode waves does not occur until the frequency is less than about $5 \mathrm{rad} \mathrm{yr}^{-1}$, and again the gravest modes (the first mode Rossby and the Kelvin waves) are the last to undergo this transition. Once the transition occurs between Rossby or Kelvin waves and their slow mode 
Coupled Waves

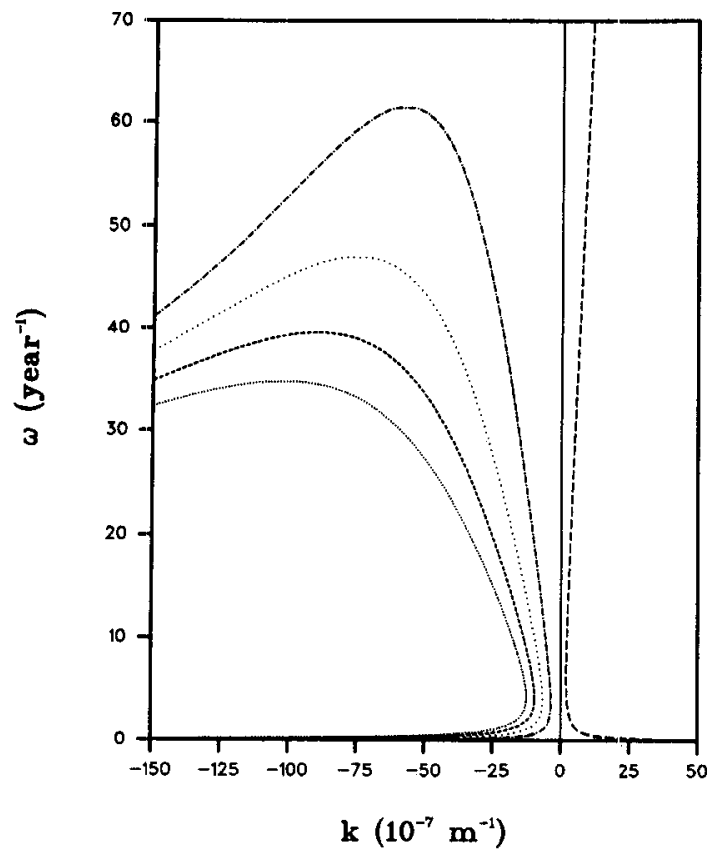

Uncoupled Waves

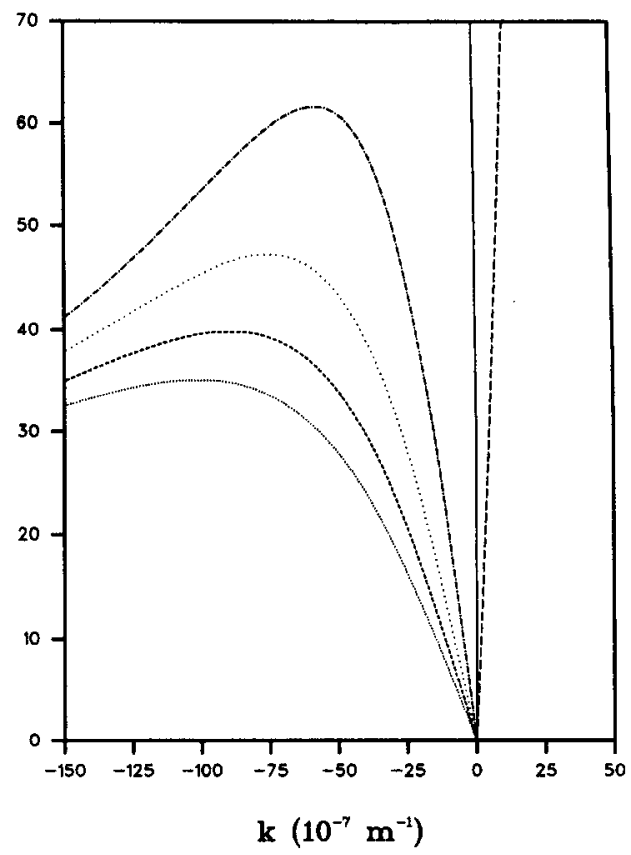

FIG. 1. A comparison between the dispersion diagrams for (a) equatorially trapped wave modes of a coupled oceanatmosphere system and (b) conventional uncoupled equatorially trapped wave modes. For the coupled waves, westward (eastward) propagating slow modes occur as low-frequency transitions from the. Rossby (Kelvin) waves. The family of curves for negative $k$ correspond to Rossby and slow wave modes $n=1$ to 4 . The dimensions for $\omega$ and $k$ follow from the parameters given in the text.

continuations, that is, at the point where $\partial k / \partial \omega=0$, the wavenumbers no longer tend toward zero with decreasing frequency. Instead the magnitude of the wavenumber increases with decreasing frequency and the group velocity reverses to be opposite in direction to the phase propagation. Also, unlike the Rossby waves, the frequency for the westward propagating slow modes increases with increasing meridional mode number.

The dispersion curves are sensitive to the parameters. Increasing $\mu$ increases the frequency at which the coupling effects become evident. Figures $2 \mathrm{a}$ and $2 \mathrm{~b}$ compare the dispersion diagrams for the cases in which $\sigma$ $=0$ and $\eta=0$, respectively, with all of the other parameters the same as in Fig. 1a. With $\sigma=0$ there is no transition to the slow mode waves, whereas with $\eta$ $=0$ these transitions do occur. This implies that the slow modes are dependent upon those ocean processes that give rise to horizontal divergence and associated vertical advection and mixing, as opposed to horizontal advection in the presence of a mean zonal temperature gradient. For this reason the slow modes here are interpreted as horizontal divergence modes.

\section{Horizontal structure}

Along with modifying the dispersion relationship, ocean-atmosphere coupling largely alters the horizon- tal structure of the equatorially trapped waves described herein. This section develops these structural modifications for the coupled Rossby and Kelvin waves, along with their slow mode transitions. As was the case for the dispersion relationship, the structural modifications to the inertial-gravity and the Rossby-gravity waves are minor, so these are omitted from further discussion, although they are included within the general formulation that follows.

\section{a. General case}

Given the dispersion relationship of Eq. (2.2.11) the eigenfunctions for $v, u, h$, and $T$ are

$$
\begin{array}{r}
v(y)=C_{1} N_{n} \exp \left[-\frac{\xi^{2}}{2}\left(1-\frac{A L^{2}}{2}\right)\right] H_{n}(\xi) \\
=C_{1} N_{n} \exp \left(-\frac{y^{2}}{2 L_{R}^{2}}\right) H_{n}(y / L) \\
u(y)=\frac{i}{\omega^{2}-k^{2} c^{2}+\mu\left(\frac{H_{0} \sigma k}{\omega}-\eta\right)} \\
\times\left[\omega \beta y v+\left(\frac{H_{0} \mu \sigma}{\omega}-k c^{2}\right) \frac{d v}{d y}\right]
\end{array}
$$




$$
\begin{aligned}
& h(y)=\frac{i H_{0}}{\omega^{2}-k^{2} c^{2}+\mu\left(\frac{H_{0} \sigma k}{\omega}-\eta\right)} \\
& \times\left[k \beta y v-\frac{\left(\omega^{2}-\mu \eta\right)}{\omega} \frac{d v}{d y}\right] \\
& T(y)=\frac{1}{\omega^{2}-k^{2} c^{2}+\mu\left(\frac{H_{0} \sigma k}{\omega}-\eta\right)} \\
& \times\left[\left(\eta-\frac{H_{0} \sigma k}{\omega}\right) \beta y v+\left(H_{0} \sigma-\frac{k c^{2} \eta}{\omega}\right) \frac{d v}{d y}\right] .
\end{aligned}
$$

Making use of the recurrence formulas for the Hermite polynomials,

$$
\begin{gathered}
\frac{d H_{n}(\xi)}{d \xi}=2 n H_{n-1}(\xi) \\
H_{n+1}(\xi)=2 \xi H_{n}(\xi)-2 n H_{n-1}(\xi),
\end{gathered}
$$

and defining

$$
\psi_{n}(\xi) \equiv N_{n} \exp \left[-\frac{\xi^{2}}{2}\left(1-\frac{A L^{2}}{2}\right)\right] H_{n}(\xi),
$$

we can obtain the following relationships:

$$
\begin{aligned}
\frac{d \psi_{n}(\xi)}{d \xi}=\left(1+\frac{A L^{2}}{2}\right) \xi \psi_{n}(\xi) & \\
& -\sqrt{2(n+1)} \psi_{n+1}(\xi) \\
\psi_{n+1}(\xi)=\left(\frac{2}{n+1}\right)^{1 / 2} & \xi \psi_{n}(\xi) \\
& -\left(\frac{n}{n+1}\right)^{1 / 2} \psi_{n-1}(\xi),
\end{aligned}
$$

which are used to express the eigenfunctions of Eqs. (3.1.1-4) in the more convenient forms:

$$
\begin{aligned}
& v(y)=C_{1} \psi_{n}(\xi) \\
& u(y)=\frac{i C_{1}}{\omega^{2}-k^{2} c^{2}+\mu\left(\frac{H_{0} \sigma k}{\omega}-\eta\right)}\left[\left(\omega \beta L-\frac{\left(H_{0} \mu \sigma-\omega k c^{2}\right)\left(2-A L^{2}\right)}{2 L \omega}\right)\left(\frac{n+1}{2}\right)^{1 / 2} \psi_{n+1}(\xi)\right. \\
& \left.+\left(\omega \beta L+\frac{\left(H_{0} \mu \sigma-\omega k c^{2}\right)\left(2+A L^{2}\right)}{2 L \omega}\right)\left(\frac{n}{2}\right)^{1 / 2} \psi_{n-1}(\xi)\right], \\
& h(y)=\frac{i C_{1} H_{0}}{\omega^{2}-k^{2} c^{2}+\mu\left(\frac{H_{0} \sigma k}{\omega}-\eta\right)}\left[\left(k \beta L+\frac{\left(\omega^{2}-\mu \eta\right)\left(2-A L^{2}\right)}{2 L \omega}\right)\left(\frac{n+1}{2}\right)^{1 / 2} \psi_{n+1}(\xi)\right. \\
& \left.+\left(k \beta L-\frac{\left(\omega^{2}-\mu \eta\right)\left(2+A L^{2}\right)}{2 L \omega}\right)\left(\frac{n}{2}\right)^{1 / 2} \psi_{n-1}(\xi)\right], \\
& T(y)=\frac{C_{1}}{\omega^{2}-k^{2} c^{2}+\mu\left(\frac{H_{0} \sigma k}{\omega}-\eta\right)}\left[\left(\frac{\beta L\left(\omega \eta-H_{0} \sigma k\right)}{\omega}-\frac{\left(H_{0} \sigma \omega-k c^{2} \eta\right)\left(2-A L^{2}\right)}{2 L \omega}\right)\left(\frac{n+1}{2}\right)^{1 / 2} \psi_{n+1}(\xi)\right. \\
& \left.+\left(\frac{\beta L\left(\omega \eta-H_{0} \sigma k\right)}{\omega}+\frac{\left(H_{0} \sigma \omega-k c^{2} \eta\right)\left(2+A L^{2}\right)}{2 L \omega}\right)\left(\frac{n}{2}\right)^{1 / 2} \psi_{n-1}(\xi)\right] .
\end{aligned}
$$

Two different scales, $L$ and $L_{R}$, appear in the eigenfunctions. The scale $L$ nondimensionalizes the meridional coordinate $y$, and $L_{R}$ is the $e$-folding scale. For the case of uncoupled waves $(\mu=0), L$ and $L_{R}$ are the same, constant, and equal to the radius of deformation for conventional equatorially trapped waves. For coupled waves, however, these scales differ from one another and they are both functions of frequency as well as the coupling and thermodynamic parameters. Using the same parameters as in Fig. $1 \mathrm{a}\left(H_{0}=200 \mathrm{~m}, c=2\right.$ $\mathrm{m} \mathrm{s}^{-1}, \beta=2.29 \times 10^{-11} \mathrm{~m}^{-1} \mathrm{~s}^{-1}, \sigma=5.0 \times 10^{-9}$ $\mathrm{K} \mathrm{m}^{-1} \mathrm{~s}^{-1}$, and $\eta=-5.0 \times 10^{-7} \mathrm{~K} \mathrm{~m}^{-1}$ ), the frequency dependencies of $L$ and $L_{R}$ are shown in Figs. 3a and
$3 \mathrm{~b}$, respectively, for different values of the coupling coefficient $\mu$, with the solid lines corresponding to $\mu$ $=0.85 \times 10^{-7} \mathrm{~m} \mathrm{~s}^{-2} \mathrm{~K}^{-1}$ (as in Fig. 1a). Both $L$ and $L_{R}$ increase with decreasing frequency from their highfrequency limit of $(c / \beta)^{1 / 2}$. This change in meridional scale is sensitive to the coupling coefficient. Increasing the coupling coefficient increases the frequency at which the coupling effects become important. These variations in the length scales parallel the behavior of the dispersion relationship wherein the coupled and conventional equatorially trapped waves differ only at low frequency. Since these length scales set the horizontal structure of the eigenfunctions, an important 
$\sigma=0$

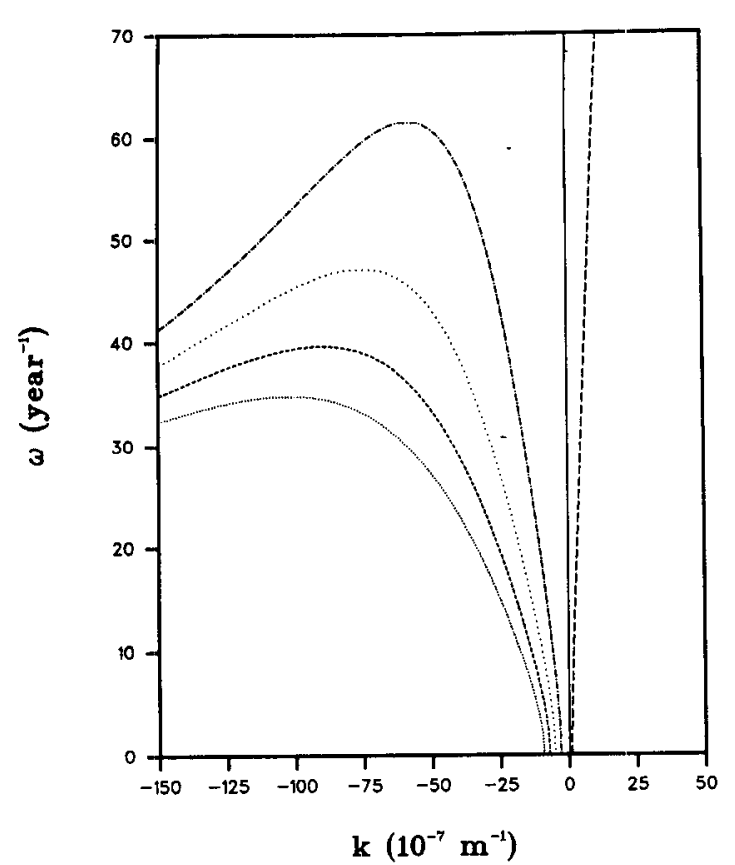

$\eta=0$

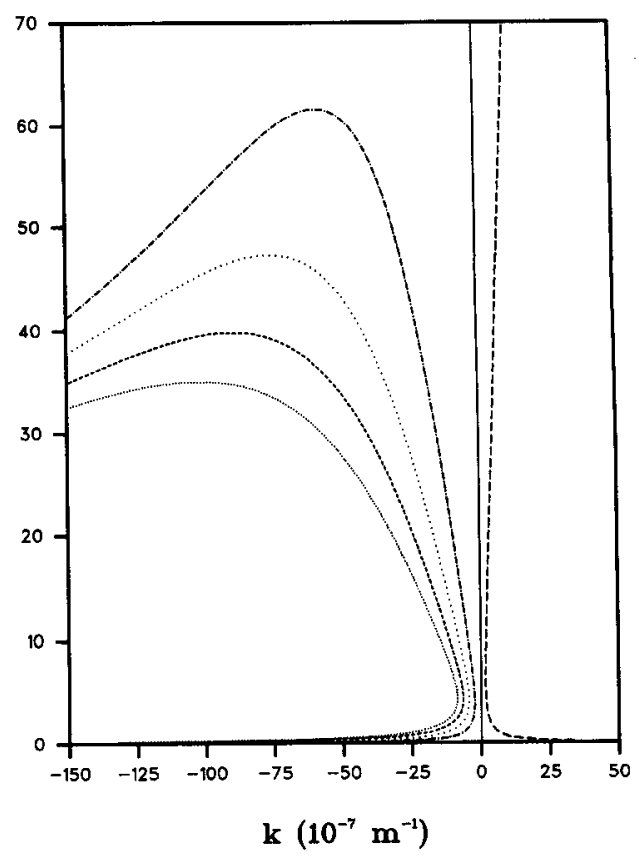

FIG. 2. As in Fig. la but for the cases in which (a) the warming coefficient $\sigma=0$ and (b) the mean zonal temperature gradient $\eta=0$.
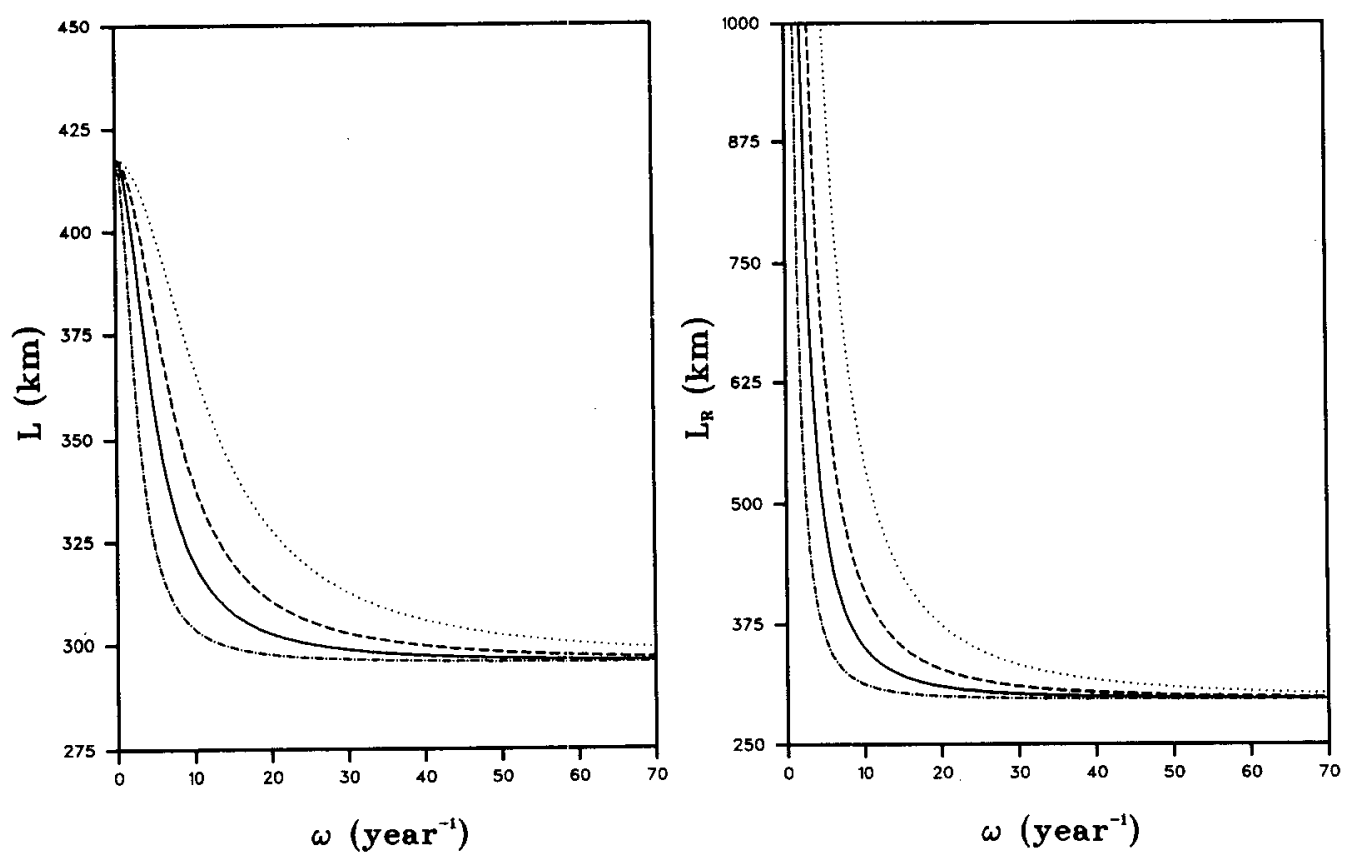

FIG. 3. The meridional scales (a) $L$ and (b) $L_{R}$ as a function of the frequency for different values of the coupling coefficient. The dotted-dashed, solid, dashed, and dotted lines are for coupling coefficients $\mu=0.25 \times 10^{-7} \mathrm{~m} \mathrm{~s}^{-2} \mathrm{~K}^{-1}$, $\mu=0.85 \times 10^{-7} \mathrm{~m} \mathrm{~s}^{-2} \mathrm{~K}^{-1}, \mu=1.92 \times 10^{-7} \mathrm{~m} \mathrm{~s}^{-2} \mathrm{~K}^{-1}$, and $\mu=5.0 \times 10^{-7} \mathrm{~m} \mathrm{~s}^{-2} \mathrm{~K}^{-1}$, respectively, with the solid line corresponding to the value used in all other plots. 
effect of the coupling is to broaden the meridional extent of the low-frequency waves. When the warming coefficient $\sigma$ is set equal to zero, $L_{R}$ equals $L$ and $L_{R}$ is somewhat smaller than for nonzero values of $\sigma$, as shown Fig. 4. Curiously, when $\eta$ is set equal to zero, $L$ decreases to zero with decreasing frequency while $L_{R}$ increases to large values, as shown in Figs. 5a and $5 \mathrm{~b}$. Despite the decreasing $L$, the meridional length scale for such coupled waves still exceeds that of the uncoupled waves because of $L_{R}$.

The broadening of the meridional length scale for low-frequency coupled equatorially trapped waves follows from the wind stress curl induced by the coupling. Without wind stress curl in the vorticity equation, the meridional component of velocity must tend to zero at low frequency, leaving the intrinsic $\beta$-plane radius of deformation as the meridional scale. With wind stress curl, the meridional component of velocity adjusts to a slowly varying Sverdrup balance, thereby broadening the meridional scale. Thus, at low frequency, the meridional scale for coupled ocean-atmosphere equatorial waves tends toward the scale of the overlying atmosphere, which in this model is imposed by the ocean.

\section{b. Gravest mode Rossby and westward propagating slow wave modes}

Given the scale dependencies on the frequency and the parameters, we examine the effects of varying scales on the eigenfunctions. Three sets of comparisons are made between the gravest mode coupled and uncoupled Rossby waves. These comparisons are for 1) Rossby waves at the frequencies $\omega=12.6 \mathrm{yr}^{-1}$, $6.28 \mathrm{yr}^{-1}$, and $2.5 \mathrm{yr}^{-1}$ (approximate periodicities of $6 \mathrm{mo}, 1 \mathrm{yr}$, and $2.5 \mathrm{yr}$, respectively), using the standard set of parameters as in Fig. 1a; 2) Rossby waves at $\omega$ $=2.5 \mathrm{yr}^{-1}$ for the extreme parameter choice of $\sigma=0$; and 3) Rossby waves at $\omega=2.5 \mathrm{yr}^{-1}$ for the extreme parameter choice of $\eta=0$. The $\omega=2.5 \mathrm{yr}^{-1}$ frequency corresponds to the transition point between coupled equatorially trapped Rossby waves and westward propagating slow mode waves. A similar set of comparisons is developed in the next section for the Kelvin waves.

The thermocline height and the horizontal velocity vector anomalies for the ocean-atmosphere coupled and the conventional equatorially trapped, gravest mode $(n=1)$ Rossby waves at the frequency $\omega$ $=12.6 \mathrm{yr}^{-1}$ are compared in Fig. 6. The meridional and zonal dimensions are in latitude and longitude degrees, respectively, with the zonal dimension spanning one wavelength. Qualitatively, both waves appear very similar, with strong zonal flows confined close to the equator and with maximum height anomalies centered at about $\pm 4^{\circ}$ for the coupled Rossby wave and $\pm 3^{\circ}$ for the uncoupled Rossby wave. From Figs. 1a and $1 \mathrm{~b}$ the wavenumbers for the coupled and the un-

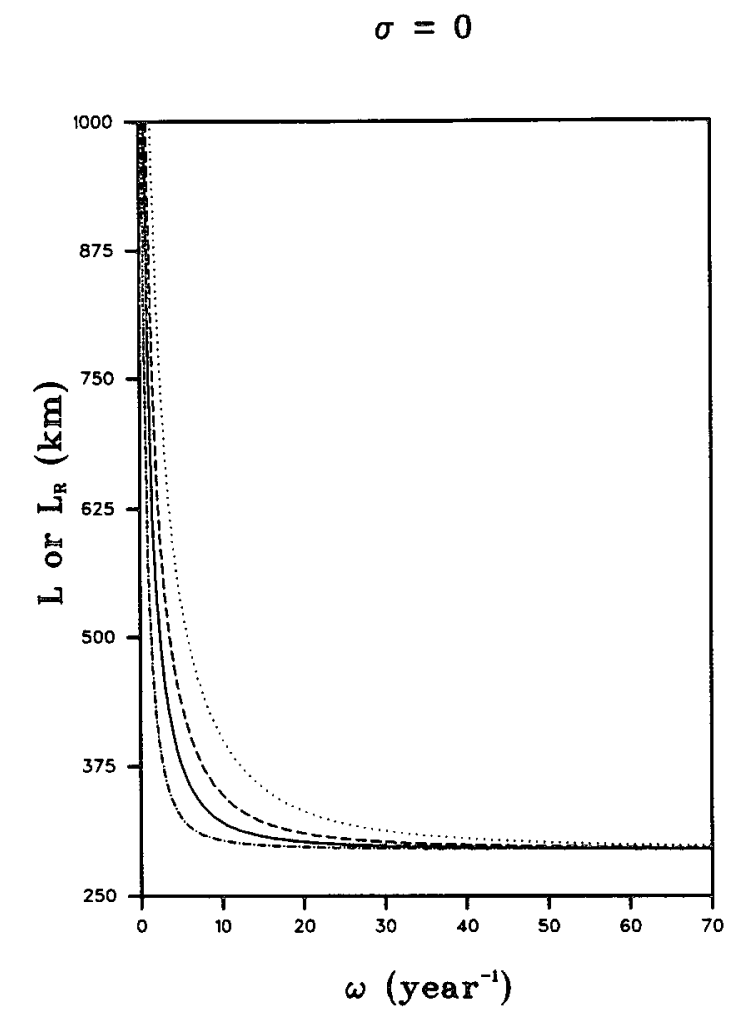

FIG. 4. As in Fig. 3 but for the case of the warming coefficient $\sigma=0$ in which $L=L_{R}$.

coupled waves at this frequency are $-6.677 \times 10^{-7} \mathrm{~m}^{-1}$ and $-6.136 \times 10^{-7} \mathrm{~m}^{-1}$, respectively, so the coupled waves have both larger meridional scale and smaller zonal scale than the uncoupled waves.

Similar presentations for the coupled and the uncoupled Rossby waves at $\omega=6.28 \mathrm{yr}^{-1}$ and for the westward propagating slow mode and the uncoupled Rossby wave at $\omega=2.5 \mathrm{yr}^{-1}$ are shown in Figs. 7 and 8 , respectively. For the uncoupled wave changing the frequency simply changes the wavelength according to the dispersion relationship (and the amplitude which is arbitrary). The effects on the coupled wave are much more noticeable. Changing the frequency from $\omega$ $=12.6 \mathrm{yr}^{-1}$ to $\omega=6.28 \mathrm{yr}^{-1}$ increases the meridional scale, which results in a poleward shift of the maximum height anomaly to about $\pm 5^{\circ}$ and a poleward broadening of the equatorial zonal jet. Qualitatively, the coupled and the uncoupled Rossby waves at $\omega$ $=6.28 \mathrm{yr}^{-1}$ are now distinctively different. A further decrease in frequency to the slow-mode transition point at $\omega=2.5 \mathrm{yr}^{-1}$ further accentuates the structural differences between this coupled mode and its counterpart uncoupled Rossby wave. The uncoupled Rossby wave shows a further increase in zonal wavelength to the extent of no longer fitting within any realistic bounded ocean basin, while the coupled mode has a much broader meridional extent, with the maximum height 
$\eta=0$

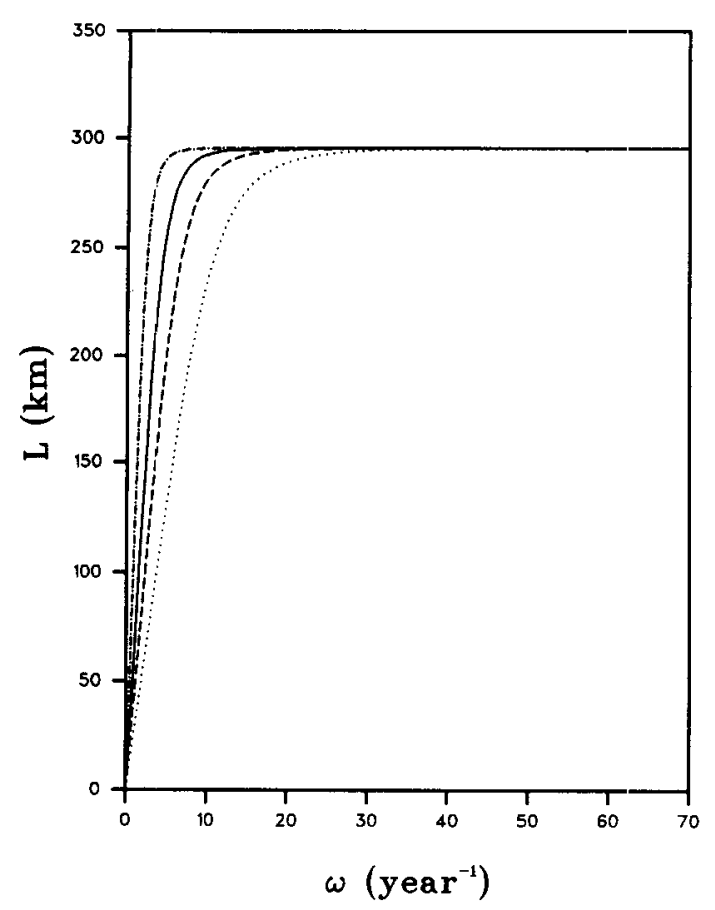

$\eta=0$

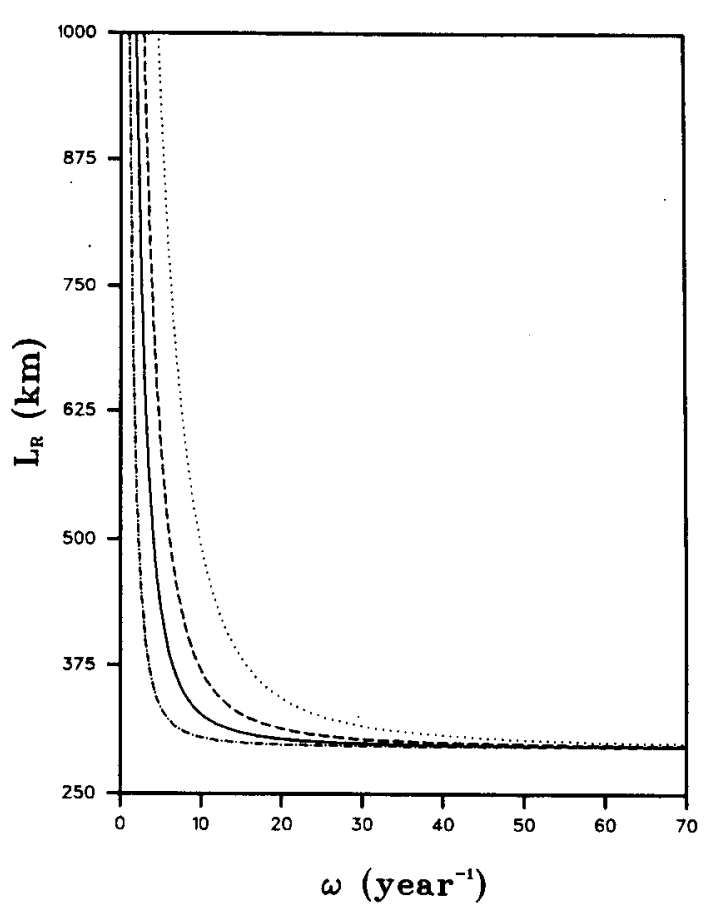

FIG. 5. As in Fig. 3 but for the case of the mean zonal temperature gradient $\eta=0$.

anomalies centered at about $\pm 10^{\circ}$ and with the zonal jet expanding to become a tropical, rather than just an equatorial feature. A further decrease in frequency for the slow-mode wave would further increase its meridional dimension and decrease its zonal dimension as contrasted with the uncoupled Rossby wave whose zonal dimension would continue to increase. The eigenfunctions for higher meridional mode coupled and uncoupled Rossby waves compare in a similar fashion as those for the gravest mode.

For the westward propagating slow-mode wave, choosing the thermodynamic parameters to have their extreme values of either $\sigma=0$ or $\eta=0$ changes the scales, but not the qualitative differences between the slow modes and their uncoupled Rossby wave counterparts. This is shown for the height and velocity anomaly fields in Fig. 9 and Fig. 10 for the cases of $\sigma$ $=0$ and $\eta=0$, respectively, with all of the other parameters as given in Fig. 1a. Recall that for the $\sigma=0$ case there is no distinct transition to a westward propagating slow mode, and hence the meridional scale for this case is smaller than it would be at the same frequency for nonzero $\sigma$, or for the $\eta=0$ case. For both cases, however, the meridional scale is substantially larger than that for the uncoupled Rossby waves, since the governing scale for the meridional structure is the $e$-folding scale, $L_{R}$, which is sensitive to the value of the coupling coefficient.

\section{c. Kelvin and eastward propagating slow wave modes}

Given the dispersion relationship of Eq. (2.3.5) for the special case of the equatorial Kelvin wave, the associated eigenfunctions are

$$
\begin{array}{r}
u(y)=C_{2} \frac{\omega}{k H_{0}} \exp \left[-\frac{\beta \omega}{2 k c^{2}} y^{2}\right], \\
h(y)=C_{2} \exp \left[-\frac{\beta \omega}{2 k c^{2}} y^{2}\right], \\
T(y)=C_{2} \frac{i\left(\sigma k H_{0}-\omega \eta\right)}{\omega k H_{0}} \exp \left[-\frac{\beta \omega}{2 k c^{2}} y^{2}\right] .
\end{array}
$$

Similar in format to the Rossby wave comparisons, Figs. 11, 12, and 13 compare the height and velocity anomaly eigenfunctions for the coupled and uncoupled Kelvin waves at the frequencies $\omega=12.6 \mathrm{yr}^{-1}$, $6.28 \mathrm{yr}^{-1}$, and $2.5 \mathrm{yr}^{-1}$, respectively. For the $\omega$ $=12.6 \mathrm{yr}^{-1}$ case there is very little difference between the coupled and the uncoupled waves. Meridional scale differences become noticeable for the $\omega=6.28 \mathrm{yr}^{-1}$ case, and upon the transition to the eastward propagating slow wave mode for the $\omega=2.5 \mathrm{yr}^{-1}$ case the scale differences are pronounced, with the meridional (zonal) scale being much larger (smaller). Without the modifications due to coupling, the zonal wavelengths for low-frequency Kelvin waves are much too large for 

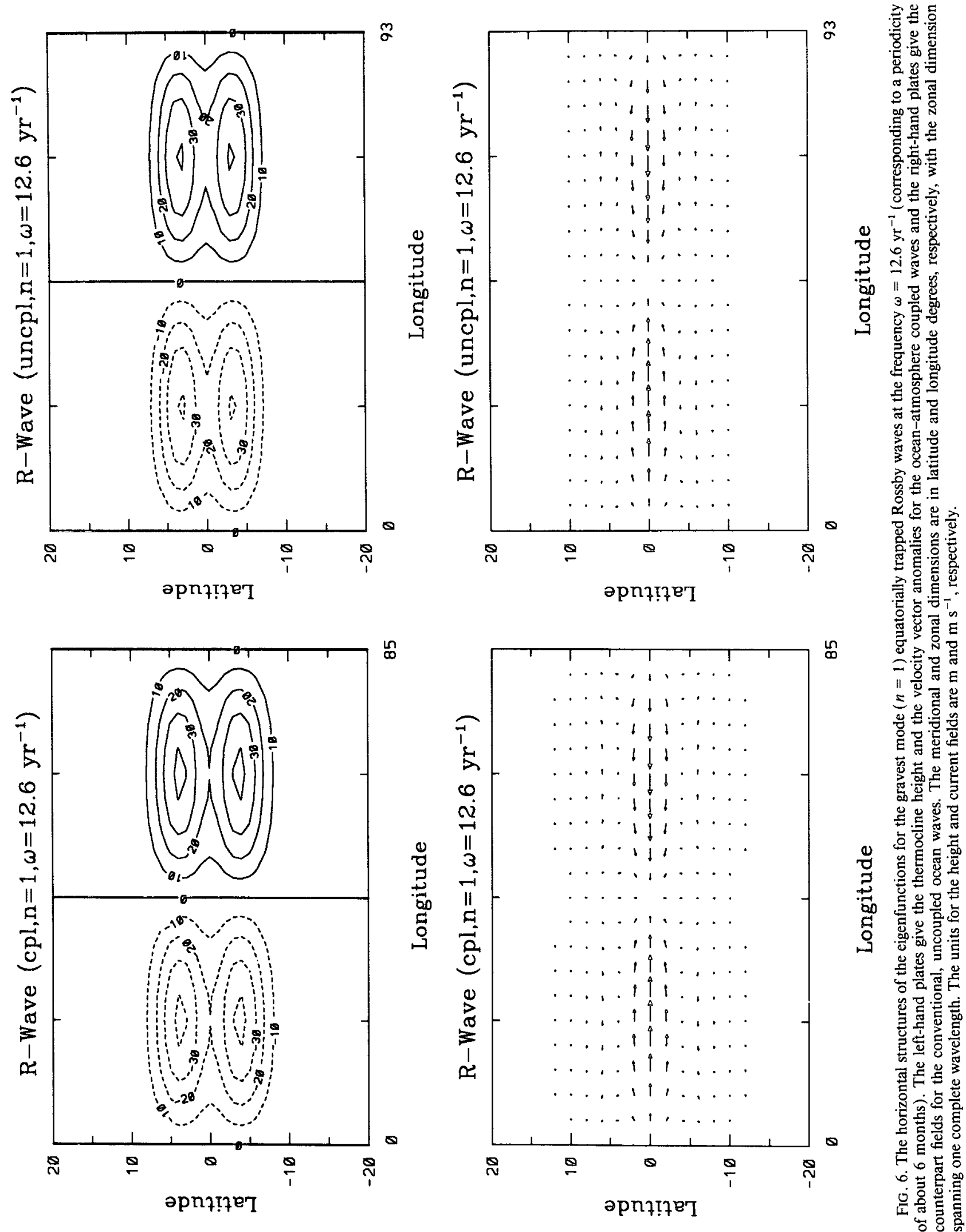

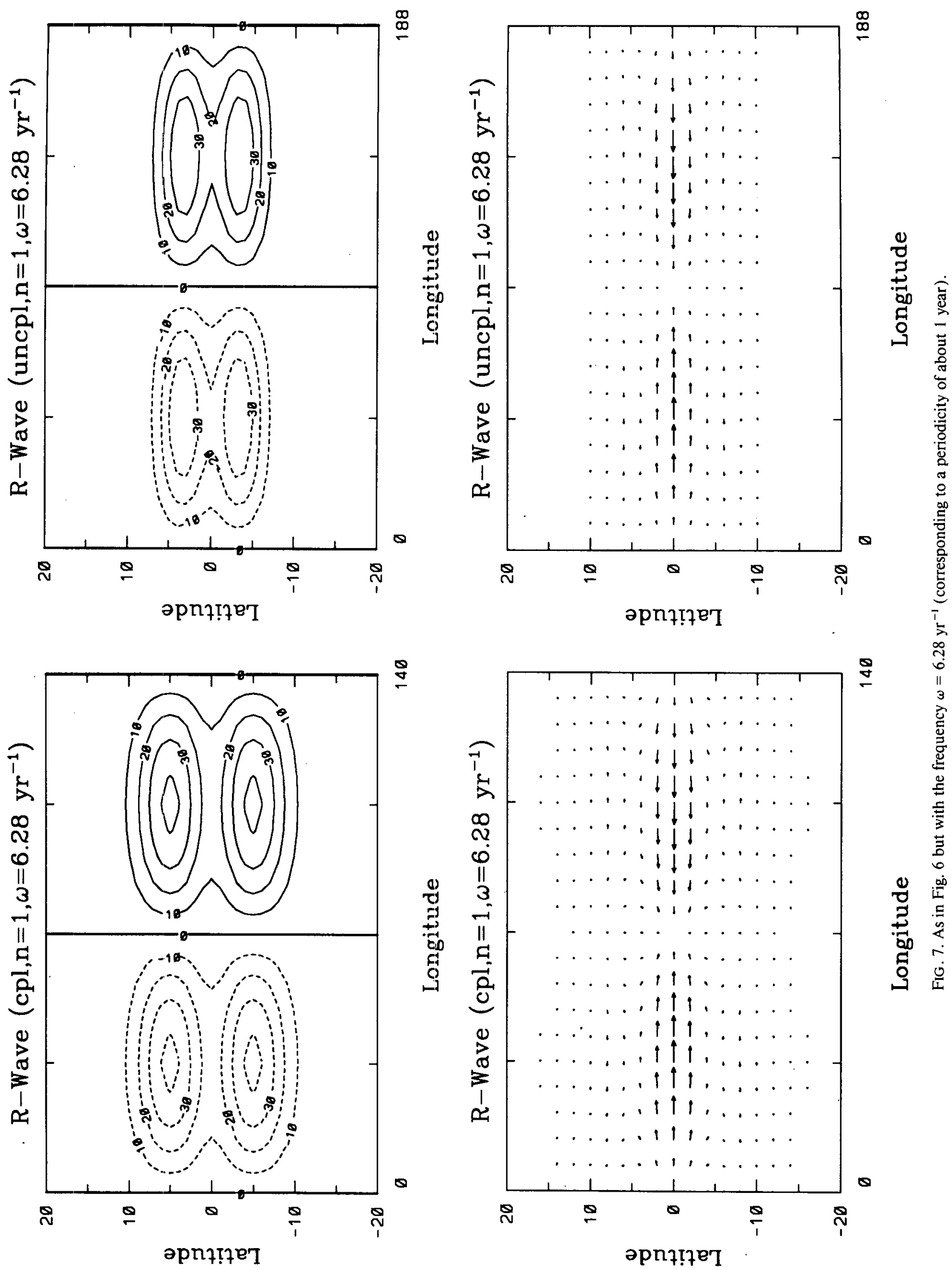

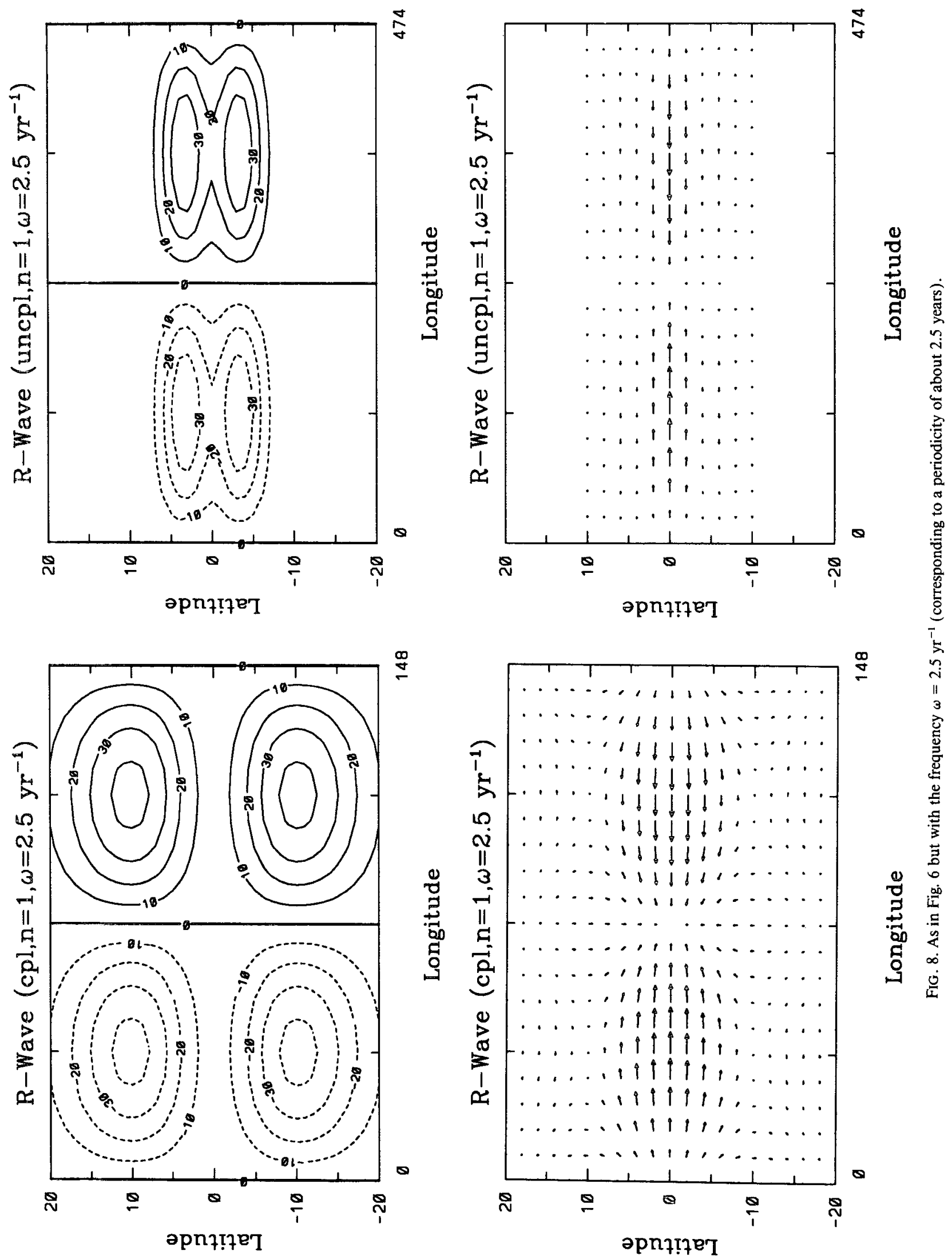

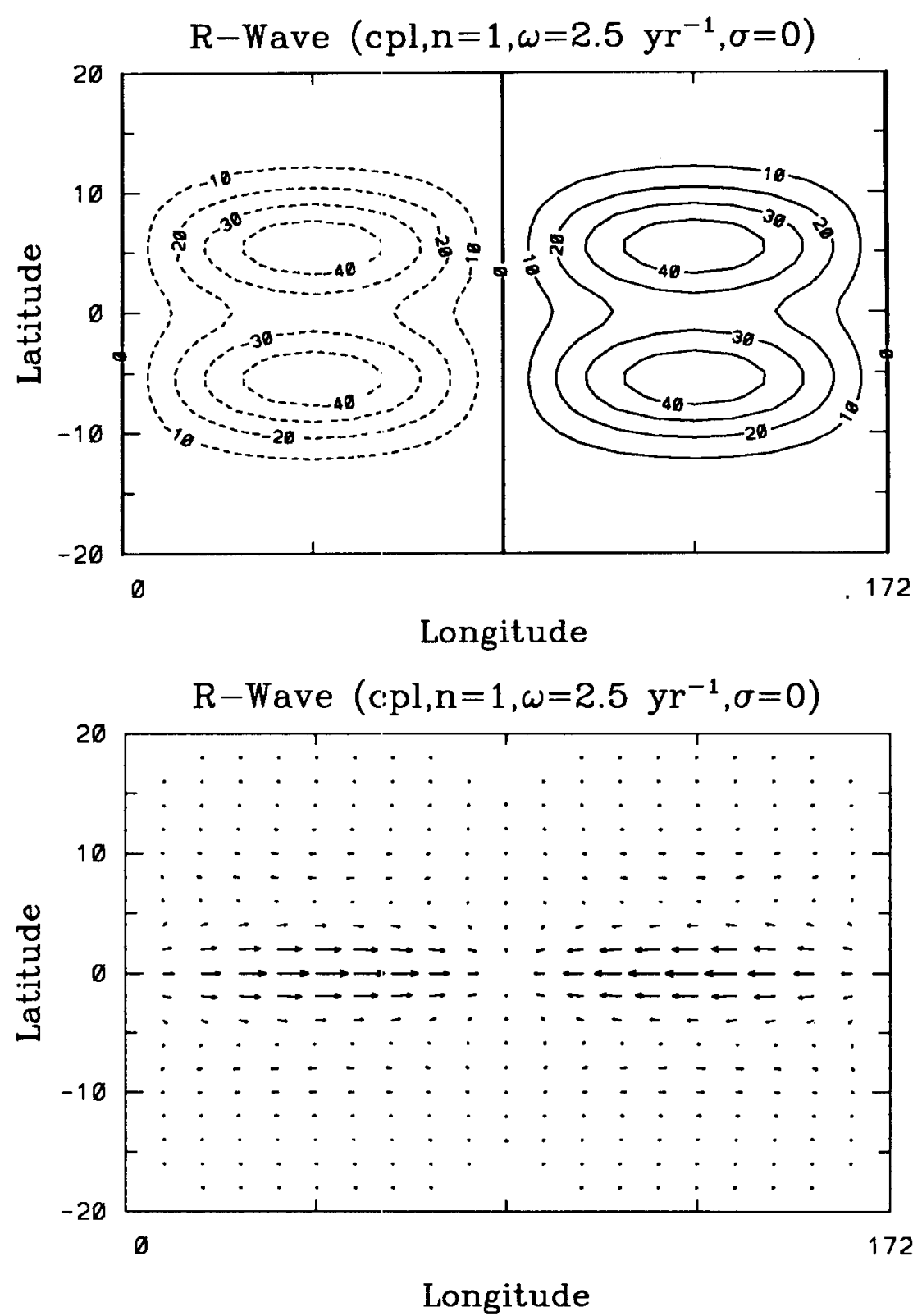

FIG. 9. The horizontal structures of the eigenfunctions for the gravest mode $(n=1)$ oceanatmosphere coupled, equatorially trapped Rossby wave at the frequency $\omega=2.5 \mathrm{yr}^{-1}$ (corresponding to a periodicity of about 2.5 years) for case of the warming coefficient $\sigma=0$. The meridional and zonal dimensions are in latitude and longitude degrees, respectively, with the zonal dimension spanning one complete wavelength. The units for the height and current fields are meters and meters per second.

these waves to fit within any realistic bounded ocean basin. The coupled Kelvin wave meridional scale, $L_{K}$ $=\left(k c^{2} / \beta \omega\right)^{1 / 2}$, has a simpler form than that for the coupled Rossby waves. Using the dispersion relationship for the coupled Kelvin wave, $L_{K}$ may be expressed as a function of frequency and the model parameters, which in the low-frequency limit becomes $L_{K}=\left(H_{0} \mu \sigma /\right.$ $\left.\beta \omega^{2}\right)^{1 / 2}$. Thus, $L_{K}$ has the same functional dependence on $\omega$ as does $L_{R}$, as shown in Fig. 14. Note that $L_{K}$ may also be expressed as $L_{K}=L_{0}[c /(\omega / k)]^{1 / 2}$, where $L_{0}=(c / \beta)^{1 / 2}$ is the ocean's equatorial Rossby radius of deformation, showing that the meridional scale for the coupled Kelvin wave is larger than that for the conventional ocean Kelvin wave since $\omega / k<c$ at low frequency. Furthermore, for the parameters of this study, as shown by Eq. (2.3.8), the phase speed for the eastward propagating slow mode depends primarily upon the coupling coefficient, the warming parameter, 

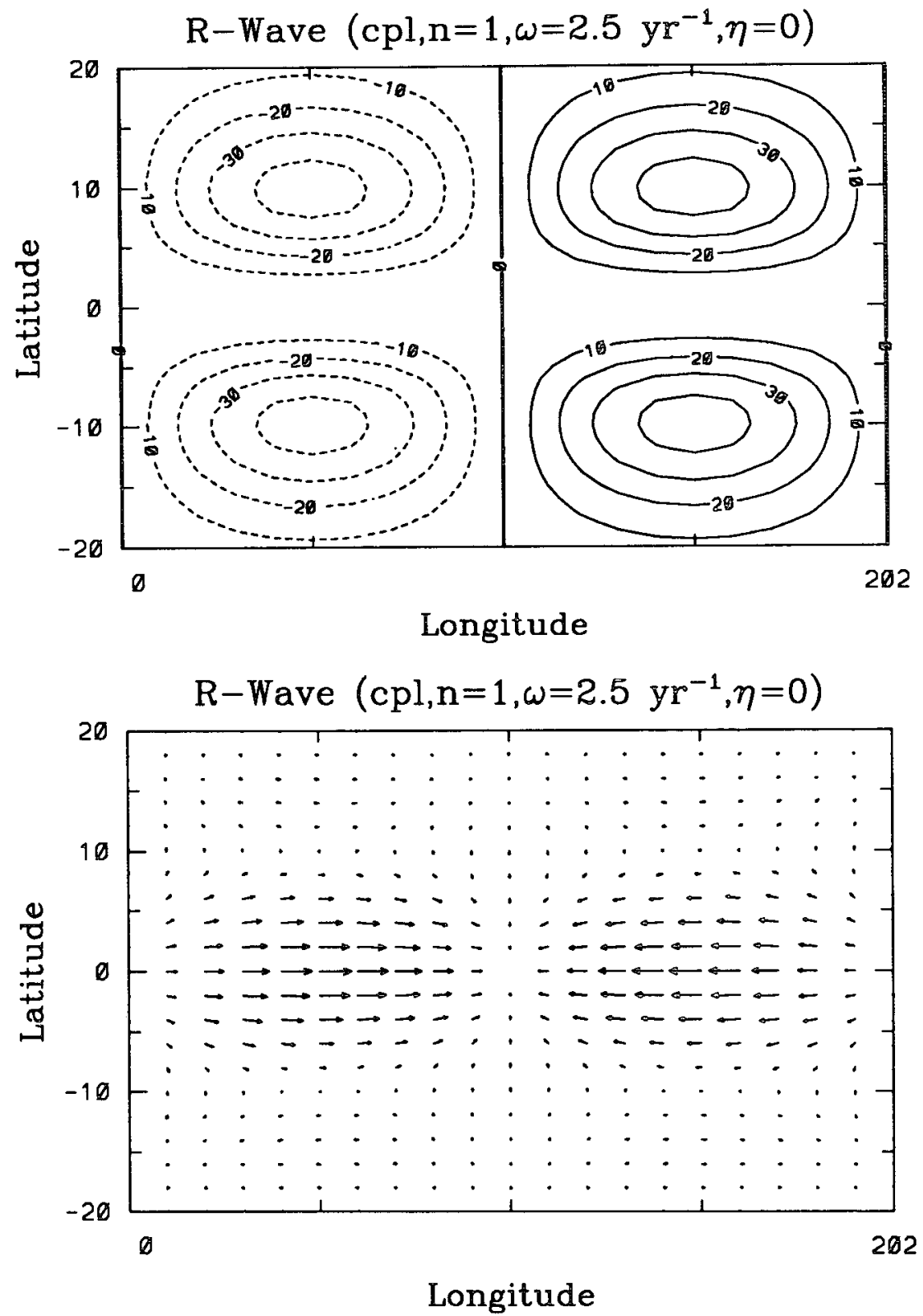

FIG. 10. As in Fig. 9 but for case of the mean zonal temperature gradient $\eta=0$.

and the background buoyancy state for the reducedgravity ocean (specified by the ocean's Kelvin wave speed); zonal advection in the presence of a mean zonal temperature gradient $\eta$ is of secondary importance.

\section{Discussion and summary}

The preceding sections described analytical solutions for equatorial wave modes of a simplified coupled ocean-atmosphere system. The simplifying assumptions, necessary to obtain a closed form solution, were those of homogeneous parameters, equal values for
Rayleigh friction, Newtonian cooling, thermal damping, and spatially uniform coupling between $\tau^{x}$ and SST. Homogeneous parameters are consistent with a model having no mixed layer formulation. The spatially variable mixed layer of the Tropics makes this assumption tenuous to the extent that perturbations owing to the included ocean dynamics are small relative to those owing to the omitted mixed layer processes. Choosing Rayleigh friction, Newtonian cooling, and thermal damping coefficients of equal value is an expediency in factoring out dissipation in the form $e^{-\gamma t}$. This facilitates a solution in terms of Hermite functions 
and results in the continuous transition between the coupled Kelvin or Rossby waves and their slow-mode counterparts. An analytical solution may also be obtained using unequal values of a lumped Rayleigh friction/Newtonian cooling parameter and a thermal damping parameter if attention is limited to just the coupled Kelvin wave and the eastward propagating slow mode (Wang and Weisberg 1994). In this case the zonal wavenumber has two roots at low frequency. The small wavenumber root approaches zero with frequency and the other root behaves like the slow mode obtained herein. For the small wavenumber root this implies that both coupling and friction (as opposed to just coupling for the other root) can supplant the intrinsic wave propagation in determining the dispersion relationship. The behavior of the solutions in the lowfrequency, low-wavenumber limit therefore depends upon how dissipation is specified. Since there is no satisfactory specification for dissipation it must be recognized that the dispersion relationship within the lowfrequency, low-wavenumber region of the dispersion plane is arbitrary. The wavenumber in this region, however, is too small to be physically realistic for a wave in a bounded basin.

The most restrictive assumption appears to be the proportionality between $\tau^{x}$ and SST. Results from numerical coupled ocean-atmosphere models that include a mixed layer formulation suggest that this assumption is good near the equator, at least when averaged over the eastern half of the Pacific Ocean (Battisti and Hirst 1989). Calculations by the present authors, using a numerical coupled ocean-atmosphere model without a mixed layer formulation, show that the correlation between zonally averaged $\tau^{x}$ and SST is maximum on the equator and diminishes to zero poleward of $14^{\circ}$ latitude. Thus, spatially uniform coupling between $\tau^{x}$ and SST is erroneous, but this weakness is tempered by the fact that the gravest mode ocean (atmosphere) equatorial waves are forced primarily by winds (heating) near the equator. Local coupling between $\tau^{x}$ and SST (without a zonal phase lag) is also erroneous since it precludes growing modes in this model. Nevertheless, despite the above limitations, the present analytical results provide insight into features of coupled model behavior that may have a more general application.

One such feature of coupled model behavior observed in our results is the increase in meridional scale at low frequency. For a more general coupled oceanatmosphere system, with separate ocean and atmosphere physics, there exist two distinctly different meridional scales determined by the intrinsic parameters of the ocean and the atmosphere. For linear perturbation, reduced-gravity ocean and atmosphere models these scales are due to the models' equivalent depths. With the atmosphere scale larger than the ocean scale, the meridional scale of the coupled oscillations tends to approach that of the atmosphere. In the present case, the intrinsic scales of the ocean and the atmosphere are the same; however, a meridional scale broadening for the coupled oscillations still occurs. A physical explanation follows from the coupling-induced wind stress curl in the model vorticity equation. With the correlation between $\tau^{x}$ and SST decreasing away from the equator, the assumption of uniform coupling may overestimate the broadening, but the broadening would occur by the wind stress curl even if the coupling and other parameters were nonuniform.

Consistent with this Sverdrup transport influence on the vorticity balance, the coupled waves differ from the uncoupled waves only over the low-frequency portion of the wave spectrum. Thus, the equatorially trapped inertial-gravity and Rossby-gravity waves are unchanged by coupling, whereas the Rossby and Kelvin waves are changed. The two primary modifications are in the dispersion relationship and in the meridional scale of the eigenfunctions. As the zonal wavenumber decreases and then as the frequency tends toward zero, the coupled Rossby and Kelvin waves show a continuous transition in the dispersion plane from short waves, nearly indistinguishable from their uncoupled counterparts, to modified long Rossby and Kelvin waves, which then transform to westward or eastward propagating slow-mode waves, respectively. Unlike conventional equatorially trapped waves, these slowmode waves have zonal wavenumber increasing with decreasing frequency, and for the westward propagating slow modes at a given zonal wavenumber, frequency increases with increasing meridional mode number. Along with the dispersion relationship, the meridional scale for the coupled ocean-atmosphere waves is a function of the model thermodynamic parameters and frequency, increasing with decreasing frequency.

Of the thermodynamic parameters, the coupling coefficient and the warming parameter are the most important ones; horizontal advection of the mean temperature gradient is of secondary importance. Upon omitting the warming parameter, which embodies the effects of horizontal divergence and vertical mixing on SST, the dispersion relationship does not show a transition to the slow mode waves, nor is the increase in meridional scale as large. As a dynamical parameter, the ocean Kelvin wave speed is also important since it sets the background buoyancy state thereby affecting the ocean divergence. The slow mode waves herein are therefore interpreted as directly forced, slowly varying oscillations feeding back to the atmosphere via the induced ocean divergence.

For the parameter values chosen, the modifications to the Kelvin and Rossby waves by the ocean-atmosphere coupling become noticeable at periodicities exceeding 6 months. Thus, the intraseasonal Kelvin waves observed in the Pacific (e.g., McPhaden and Taft 1988; Johnson and McPhaden 1993) and the superposition of Kelvin and Rossby waves describing the seasonal evolution of the equatorial thermocline in the 

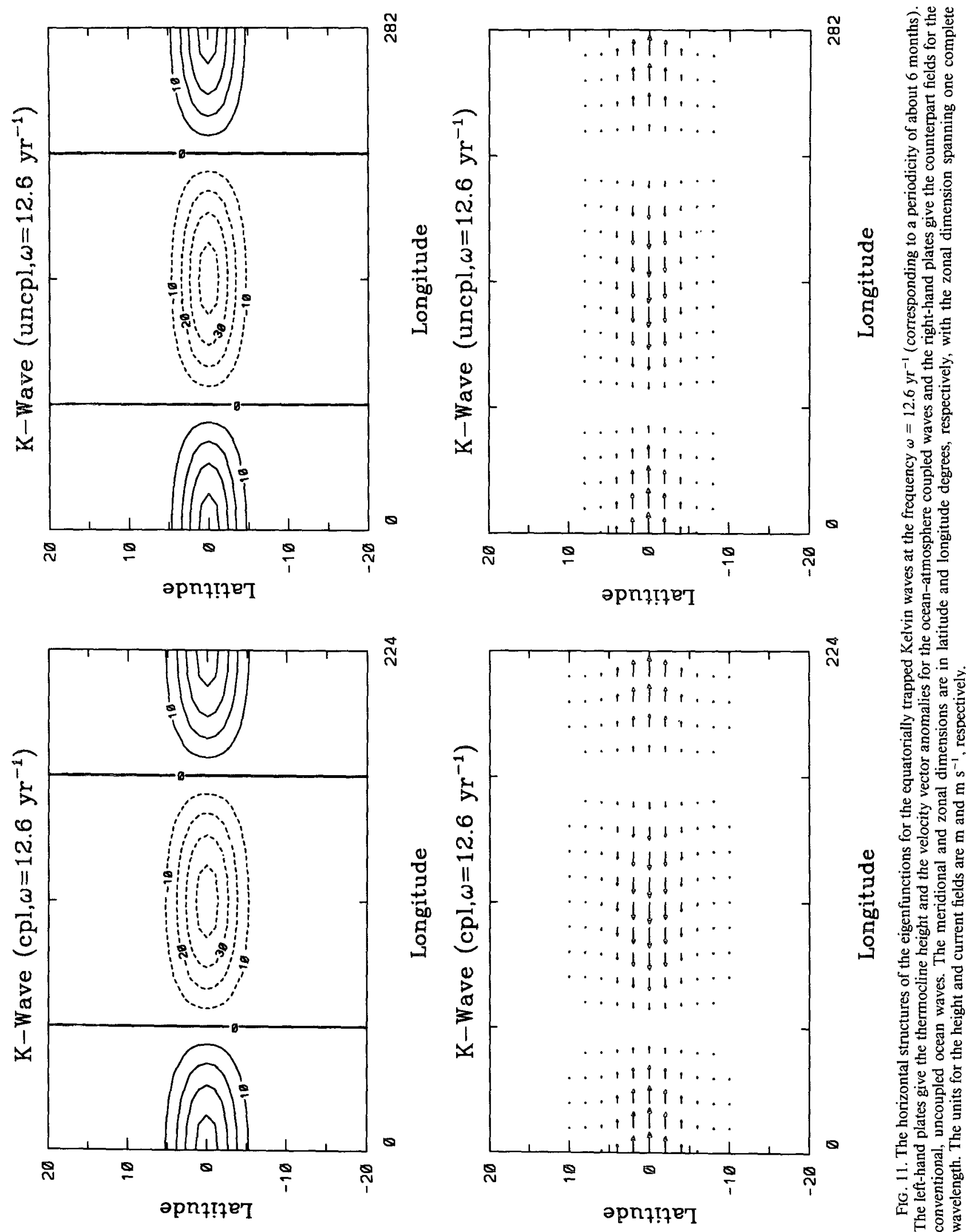

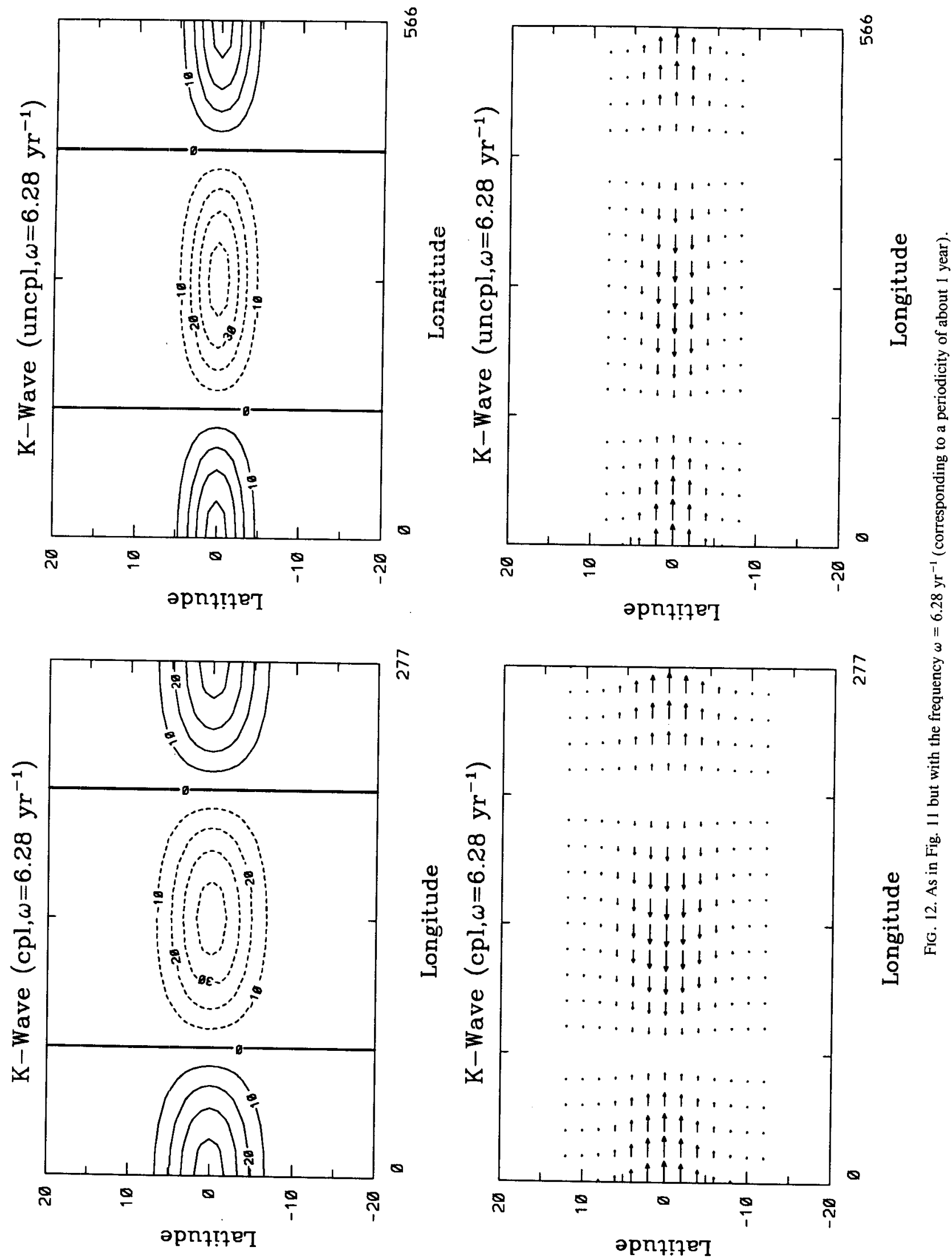

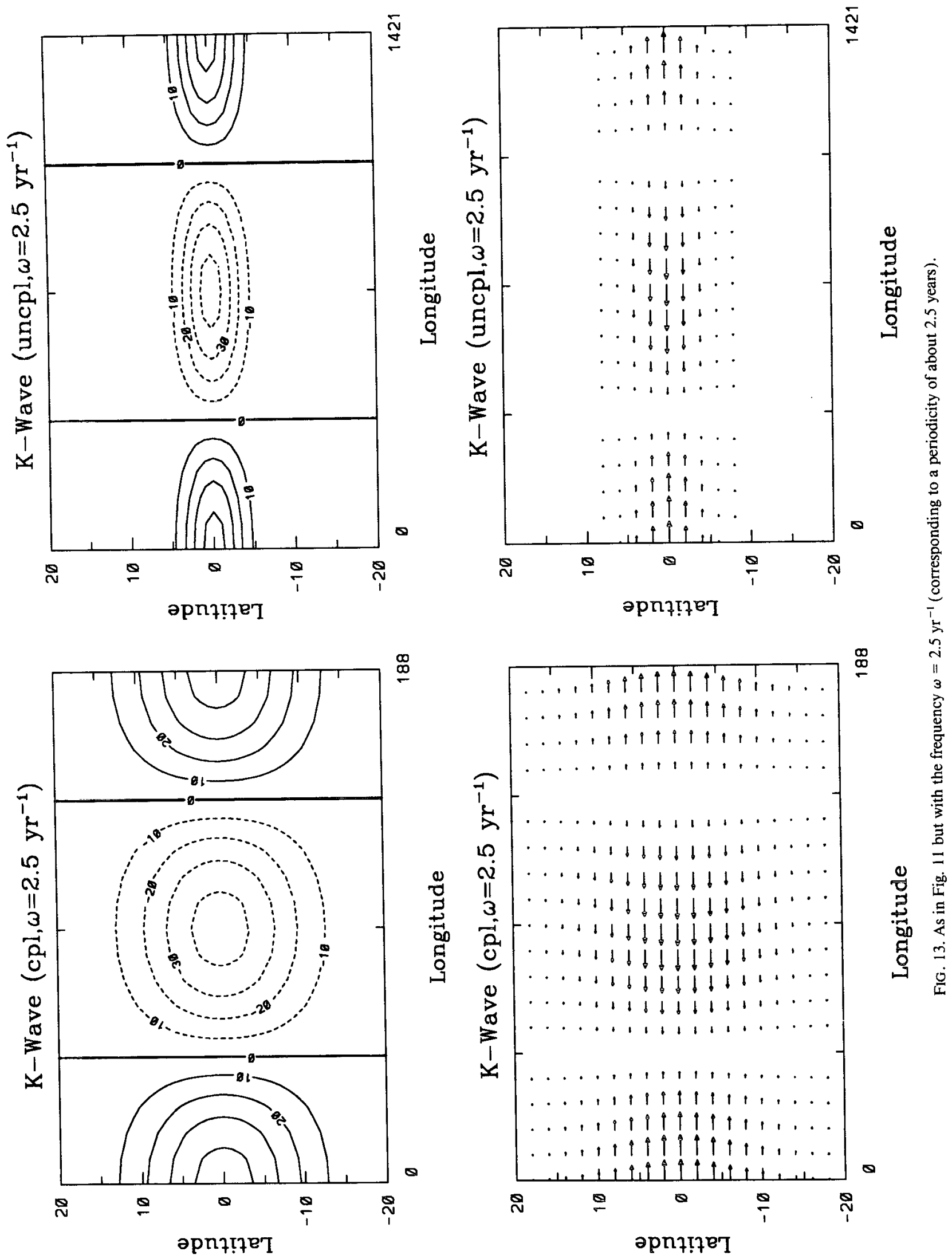


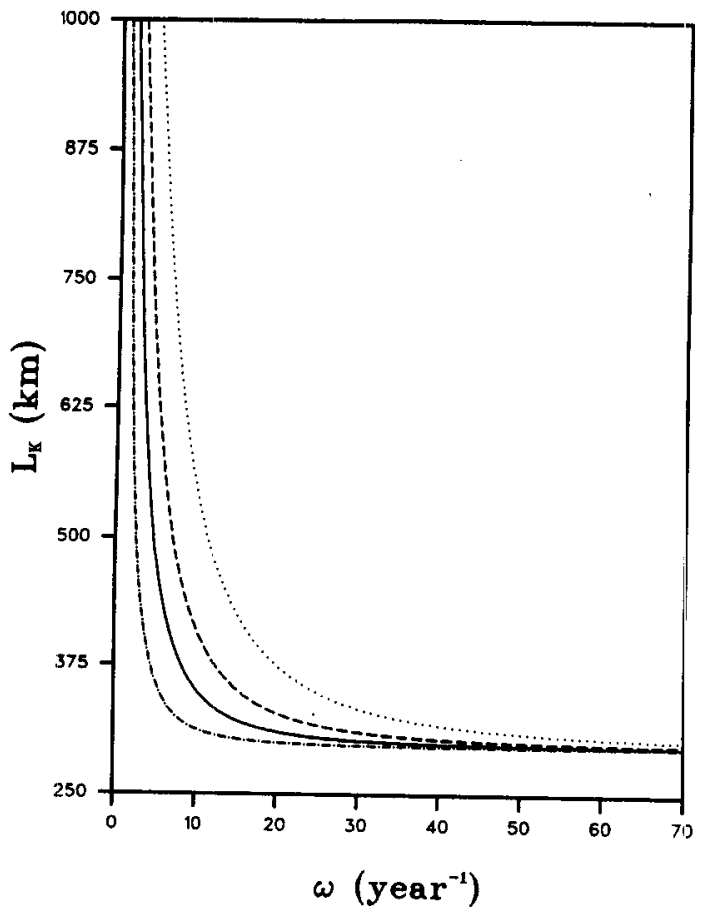

FIG. 14. The $e$-folding scale $L_{K}$ for the ocean-atmosphere coupled Kelvin wave as a function of the frequency for different values of the coupling coefficient. The dotted-dashed, solid, dashed, and dotted lines correspond to the coupling coefficient values $\mu=0.25 \times 10^{-7}$ $\mathrm{m} \mathrm{s}^{-2} \mathrm{~K}^{-1}, \mu=0.85 \times 10^{-7} \mathrm{~m} \mathrm{~s}^{-2} \mathrm{~K}^{-1}, \mu=1.92 \times 10^{-7} \mathrm{~m} \mathrm{~s}^{-2} \mathrm{~K}^{-1}$, and $\mu=5.0 \times 10^{-7} \mathrm{~m} \mathrm{~s}^{-2} \mathrm{~K}^{-1}$, respectively, with the solid line corresponding to the value used in the previous plots.

Atlantic (e.g., Weisberg and Tang 1987, 1990) appear to be correctly characterized by conventional equatorially trapped wave theory. However, for periodicities of a year or longer, the modifications due to the oceanatmosphere coupling suggest that conventional trapped wave theory may no longer be applicable. This is supported by the Philander et al. (1992) high-resolution coupled GCM experiment wherein they find no evidence for ocean Kelvin and Rossby waves on interannual timescales, whereas these waves are prominent on shorter timescales. The structural differences between the coupled waves and their conventional equatorially trapped wave counterparts also provide for speculation on why the low- and high-resolution coupled GCM experiments of Lau et al. (1992) and Fhilander et al. (1992), respectively, arrive at similar El Niño and La Niña states, but in different ways. The high-resolution model can resolve all of the equatorial waves, whereas the low-resolution model can only resolve broader meridional-scale slow modes.

Given the coupled wave modifications, the question of the importance of so-called off-equatorial Rossby waves for ENSO may be reexamined. In a series of communications summarized by Graham and White (1991), oceanic processes occurring poleward of the equatorial waveguide (defined by a multiple of the ocean's equatorial Rossby radius of deformation ) are argued as being important elements for establishing the character of the ENSO cycle. Specifically, it is argued that 1) the meridional scale for ENSO variability exceeds that of the oceanic waveguide, 2) this scale broadening is related to Ekman pumping, and 3) the Ekman pumping, in turn, is related to equatorial SST. Increased meridional scale and the importance of Ekman pumping has been inferred from historical temperature data. Kessler (1990) found that the region of maximum vertical displacement for the $20^{\circ} \mathrm{C}$ isotherm, during the period $1970-1987$, was at $12^{\circ} \mathrm{N}$ near the western boundary and that the progression of off-equatorial Rossby waves during the 1972 El Niño was related to the wind stress curl distribution associated with equatorial westerly wind anomalies. Where controversy has ensued is with the importance of these off-equatorial Rossby waves upon reflection at the western boundary and subsequent eastward propagation as Kelvin waves. Kessler (1991), Battisti (1991), and Wakata and Sarachik (1991) argue that the equatorial Kelvin wave results primarily from the reflection of the gravest mode Rossby wave, leading the former two authors to define off-equatorial as the region poleward of $\pm 8^{\circ}$ and concluding, based upon western boundary reflection arguments, that off-equator variations should not be a major factor in ENSO. In contrast, Graham and White (1991) contend that coupled model simulations of ENSO are greatly altered if effects from poleward of $\pm 8^{\circ}$ are neglected. The result of increased meridional scale for equatorially trapped waves of a coupled ocean-atmosphere system, owing to wind stress curl, is consistent with the findings of both groups of investigators. With the meridional scale of the coupled modes increasing beyond that of the oceanic radius of deformation, off-equator variability becomes important, but for reasons other than conventional equatorial wave reflection. In this sense our results agree with the conclusion of Battisti (1991) that "an argument for a significant role for the off-equatorial signals in the ENSO cycle must incorporate exotic effects." These effects may not be exotic; they may simply reflect the interactive forcing between the ocean and the atmosphere occurring slowly at low frequency.

Even in the simplest ocean-atmosphere coupled, linear perturbation reduced-gravity models (e.g., Hirst 1988; Wang and Weisberg 1994) the evolving spatial structures appear more Kelvin-like (Rossby-like) in the eastern (western) half of the ocean basin. A single Kelvin-like or Rossby-like wave mode cannot describe these results. For example, the evolving structure across the basin of the Hirst (1988) U1 mode is different from the eastward propagating slow mode herein. At the very least, a wave interpretation would require a superposition of waves with different meridional structures, consistent with a forced wave problem. How these waves might reflect at the ocean boundaries, however, 
is conceptually difficult since the ocean is bounded while the atmosphere is not.

In summary, solutions have been presented for equatorially trapped waves of a simplified coupled ocean-atmosphere system. The effects of coupling are found to be frequency dependent. Inertial-gravity and Rossby-gravity waves are not modified, whereas the Kelvin and Rossby waves are, and as frequency tends toward zero these latter waves transform into slow eastward or westward propagating modes, respectively. The transition region from conventional to slow mode waves occurs where the intrinsic dispersion properties of the ocean medium are supplanted by the coupling between the ocean and the atmosphere. For the parameters used, the modifications by coupling become noticeable at periodicities longer than 6 months. The primary modifications are a decrease in phase speed and an increase in meridional scale. The meridional scale increase is consistent with both hydrographic observations and results from ENSO-related models, and a physical explanation follows the wind stress curl induced in the ocean's vorticity balance by the air-sea coupling. However, achieving a closed form solution necessitated several restrictive assumptions on the model thermodynamics, air-sea coupling, and dissipation. The effects of these on the general nature of the findings warrant further study.

Acknowledgments. Support for this work was provided by the Division of Ocean Sciences, National Science Foundation, Grant OCE-9100024. Constructive criticisms provided by Dr. J. McCreary and the anonymous reviewers are gratefully acknowledged.

\section{APPENDIX}

\section{Eigenfunction Orthogonality, Normalization, and Completeness}

Equation (2.2.5) can be formulated as the SturmLiouville equation:

$\frac{d}{d \xi}\left[p(\xi) \frac{d v(\xi)}{d \xi}\right]-s(\xi) v(\xi)+\lambda r(\xi) v(\xi)=0$

where

$$
\begin{gathered}
p(\xi)=\exp \left(-\frac{A L^{2}}{2} \xi^{2}\right) \\
s(\xi)=\left(D^{2} L^{4} \xi^{2}+\frac{A L^{2}}{2}-1\right) \exp \left(-\frac{A L^{2}}{2} \xi^{2}\right), \\
r(\xi)=\exp \left(-\frac{A L^{2}}{2} \xi^{2}\right)
\end{gathered}
$$

and

$$
\lambda=L^{2}\left(B+\frac{A}{2}\right)-1 .
$$

Equation (A.1) has the eigenfunctions $v_{n}(\xi) \equiv \psi_{n}(\xi)$ for the eigenvalues $\lambda_{n}$. Consider any two eigenfunctions $v_{m}$ and $v_{n}$ and the corresponding eigenvalues $\lambda_{m}$ and $\lambda_{n}$, then

$$
\frac{d}{d \xi}\left[p(\xi) \frac{d v_{m}}{d \xi}\right]-s(\xi) v_{m}+\lambda_{m} r(\xi) v_{m}=0
$$

and

$$
\frac{d}{d \xi}\left[p(\xi) \frac{d v_{n}}{d \xi}\right]-s(\xi) v_{n}+\lambda_{n} r(\xi) v_{n}=0
$$

After multiplying Eq. (A.6) by $v_{n}$, Eq. (A.7) by $v_{m}$, subtracting and integrating from $-\infty$ to $+\infty$, the terms containing $s(\xi)$ cancel and the two derivative terms may be simplified by integration by parts, one of which becomes

$$
\begin{aligned}
\int_{-\infty}^{+\infty} v_{n} & \frac{d}{d \xi}\left(p \frac{d v_{m}}{d \xi}\right) d \xi \\
& =\left.v_{n} p \frac{d v_{m}}{d \xi}\right|_{-\infty} ^{+\infty}-\int_{-\infty}^{+\infty} p \frac{d v_{m}}{d \xi} \frac{d v_{n}}{d \xi} d \xi
\end{aligned}
$$

Thus, when the two derivative terms are subtracted, the integrals on the right-hand side of Eq. (A.8) cancel out. The boundary condition that $v(y) \rightarrow 0$ as $y \rightarrow$ $\pm \infty$ also allows the first term on the right-hand side of Eq. (A.8) to vanish. The net result is thus

$$
\left(\lambda_{n}-\lambda_{m}\right) \int_{-\infty}^{+\infty} r(\xi) v_{m}(\xi) v_{n}(\xi) d \xi=0
$$

which leads to the statement that eigenfunctions corresponding to different eigenvalues are orthonormal with respect to the weight function $r(\xi)$. Therefore, we can conclude that

$$
\begin{gathered}
\int_{-\infty}^{+\infty} r(\xi) v_{m}(\xi) v_{n}(\xi) d \xi=\int_{-\infty}^{+\infty} r(\xi) \psi_{m}(\xi) \psi_{n}(\xi) d \xi \\
\left.\quad=\int_{-\infty}^{+\infty} N_{m} N_{n} e^{-\xi^{2}} H_{m}(\xi) H_{n}(\xi) d \xi=\delta_{m n}, \quad \text { (A. } 10\right)
\end{gathered}
$$

where $\delta_{m n}=1$, if $m=n$, and $\delta_{m n}=0$, if $m \neq n$.

Given the orthogonality property of the eigenfunctions, a function $f(\xi)$ may be represented by an infinite series expansion of these eigenfunctions:

$$
f(\xi)=\sum_{m} a_{m} \psi_{m}(\xi) .
$$

The expansion coefficients $a_{m}$ are obtained by multiplying both sides of (A.11) by $r(\xi) \psi_{n}(\xi)$ and integrating from $-\infty$ to $+\infty$, assuming that term-by-term integration is permissible, and employing the orthogonality 
property. From this operation, only the term with $n$ $=m$ will remain on the right-hand side, yielding

$$
\begin{aligned}
a_{n} & =\int_{-\infty}^{+\infty} f(\xi) r(\xi) \psi_{n}(\xi) d \xi \\
& =\int_{-\infty}^{+\infty} f(\xi) \exp \left[-\frac{\xi^{2}}{2}\left(1+\frac{A L^{2}}{2}\right)\right] H_{n}(\xi) d \xi
\end{aligned}
$$

\section{REFERENCES}

Barnett, T. P., M. Latif, E. Kirk, and E. Roeckner, 1991: On ENSO physics. J. Climate, 4, 487-515.

Battisti, D. S., 1991: Reply. J. Phys. Oceanogr., 21, 461-465.

$\longrightarrow$, and A. C. Hirst, 1989: Interannual variability in the tropical atmosphere-ocean model: Influence of the basic state, ocean geometry, and nonlinearity. J. Atmos. Sci., 45, 1687-17.12.

Cane, M. A., M. Munnich, and S. E. Zebiak, 1990: A study of selfexcited oscillations of the tropical ocean-atmosphere system. Part I: Linear analysis. J. Atmos. Sci., 47, 1562-1577.

Chao, Y., and S. G. H. Philander, 1993: On the structure of the Southern Oscillation. J. Climate, 6, 450-469.

Gill, A. E., 1980: Some simple solutions for heat-induced tropical circulation. Quart. J. Roy. Meteor. Soc., 106, 447-462.

Graham, N. E., and W. B. White, 1991: Comments on "On the role of off-equatorial oceanic Rossby waves during ENSO." J. Phys. Oceanogr., 21, 453-460.

Hirst, A. C., 1986: Unstable and damped equatorial modes in simple coupled ocean-atmosphere models. J. Atmos. Sci., 43, 606630 .

- 1988 : Slow instabilities in tropical ocean basin-global atmosphere models. J. Atmos. Sci., 45, 830-852.

Johnson, E. S., and M. J. McPhaden, 1993: Structure of intraseasonal Kelvin waves in the equatorial Pacific Ocean. J. Phys. Oceanogr., 23, 608-625.

Katz, E. J., 1987: Equatorial Kelvin waves in the Atlantic. J. Geophys. Res., 92, 1894-1898.

Kessler, W. S., 1990: Observations of long Rossby waves in the northern tropical Pacific. J. Geophys. Res., 95, 5183-5219.

_-_ 1991: Can reflected extra-equatorial Rossby waves drive ENSO? J. Phys. Oceanogr., 21, 444-452.
Knox, R. A., and D. Halpern, 1982: Long range Kelvin wave propagation of transport variations in Pacific Ocean equatorial current. J. Mar. Res., 40, 329-339.

Lau, K. M., 1981: Oscillation in a simple equatorial climate system. J. Atmos. Sci., 38, 248-261.

- , S. G. H. Philander, and M. J. Nath, 1992: Simulation of ENSOlike phenomena with a low-resolution coupled GCM of the global ocean and atmosphere. J. Climate, 5, 284-307.

Matsuno, T., 1966: Quasi-geostrophic motions in the equatorial area. J: Meteor. Soc. Japan, 2, 25-43.

McCreary, J. P., and D. L. T. Anderrson, 1991: An overview of coupled ocean-atmosphere models of El Niño and the Southern Oscillation. J. Geophys. Res., 96, 3125-3150.

McPhaden, M. J., and B. A. Taft, 1988: Dynamics of seasonal and intraseasonal variability in the eastern equatorial Pacific. J. Phys. Oceanogr., 18, 1713-1732.

Neelin, J. D., 1991: The slow sea surface temperature mode and the fast-wave limit: Analytic theory for tropical interannual oscillations and experiments in hybrid coupled models. J. Atmos. Sci., 48, 584-606.

Philander, S. G. H., R. C. Pacanowski, N. C. Lau, and M. J. Nath, 1992: Simulation of ENSO with a global atmosphere GCM coupled to a high-resolution, tropical Pacific Ocean GCM. J. Climate, 5, 308-329.

Schopf, P. S., and M. J. Suarez, 1990: Ocean wave dynamics and time scale of ENSO. J. Phys. Oceanogr., 20, 629-645.

Suarez, M. J., and P. S. Schopf, 1988: A delayed action oscillator for ENSO. J. Atmos. Sci., 45, 3283-3287.

Wakata, Y., and E. S. Sarachik, 1991: On the role of equatorial ocean modes in the ENSO cycle. J. Phys. Oceanogr., 21, 434-443.

Wang, C., and R. H. Weisberg, 1994: On the "slow mode" mechanism in ENSO-related coupled ocean-atmosphere models. J. Climate, 7 , in press.

Weisberg, R. H., and T. Y. Tang, 1987: Further studies on the response of the equatorial thermocline in the Atlantic Ocean to the seasonally varying trade winds. J. Geophys. Res., 92, 3709-3727.

- and - 1990: A linear analysis of equatorial Atlantic Ocean. J. Phys. Oceanogr., 20, 1813-1825.

White, W. B., S. E. Pazan, and M. Inoue, 1987: Hindcast/forecast of ENSO events based on redistribution of observed and model heat content in the western tropical Pacific, 1964-1986. J. Phys. Oceanogr., 17, 264-280.

, Y. H. He, and S. E. Pazan, 1989: Redistribution of subsurface thermal structure during the onset of the 1982-83 and 198687 ENSO events and the 1984-85 anti-ENSO event. J. Phys. Oceanogr., 19, 1397-1406. 